\title{
Weak lensing reveals a tight connection between dark matter halo mass and the distribution of stellar mass in massive galaxies
}

\author{
Song Huang ${ }^{\oplus}, 1,2,8 \star$ Alexie Leauthaud ${ }^{\odot},{ }^{1}$ Andrew Hearin, ${ }^{3}$ Peter Behroozi ${ }^{\oplus},{ }^{4}$ \\ Christopher Bradshaw, ${ }^{1}$ Felipe Ardila ${ }^{\odot},{ }^{1}$ Joshua Speagle ${ }^{\odot}, 5$ Ananth Tenneti ${ }^{\odot}, 6$ \\ Kevin Bundy, ${ }^{7}$ Jenny Greene, ${ }^{8}$ Cristóbal Sifón ${ }^{\oplus 9}$ and Neta Bahcall ${ }^{8}$ \\ ${ }^{1}$ Department of Astronomy and Astrophysics, University of California Santa Cruz, 1156 High St., Santa Cruz, CA 95064, USA \\ ${ }^{2}$ Kavli-IPMU, The University of Tokyo Institutes for Advanced Study, the University of Tokyo (Kavli IPMU, WPI), Kashiwa 277-8583, Japan \\ ${ }^{3}$ Argonne National Laboratory, Argonne, IL 60439, USA \\ ${ }^{4}$ Department of Astronomy and Steward Observatory, University of Arizona, Tucson, AZ 85721, USA \\ ${ }^{5}$ Department of Astronomy, Harvard University, 60 Garden St, MS 46, Cambridge, MA 02138, USA \\ ${ }^{6}$ McWilliams Center for Cosmology, Department of Physics, Carnegie Mellon University, Pittsburgh, PA 15213, USA \\ ${ }^{7}$ UCO/Lick Observatory, University of California, Santa Cruz, 1156 High Street, Santa Cruz, CA 95064, USA \\ ${ }^{8}$ Department of Astrophysical Sciences, Peyton Hall, Princeton University, Princeton, NJ 08540, USA \\ ${ }^{9}$ Instituto de Física, Pontificia Universidad Católica de Valparaíso, Casilla 4059, Valparaíso, Chile
}

Accepted 2019 November 8. Received 2019 November 8; in original form 2018 November 1

\begin{abstract}
Using deep images from the Hyper Suprime-Cam (HSC) survey and taking advantage of its unprecedented weak lensing capabilities, we reveal a remarkably tight connection between the stellar mass distribution of massive central galaxies and their host dark matter halo mass. Massive galaxies with more extended stellar mass distributions tend to live in more massive dark matter haloes. We explain this connection with a phenomenological model that assumes, (1) a tight relation between the halo mass and the total stellar content in the halo, (2) that the fraction of in situ and ex situ mass at $r<10 \mathrm{kpc}$ depends on halo mass. This model provides an excellent description of the stellar mass functions (SMFs) of total stellar mass $\left(M_{\star}^{\text {max }}\right)$ and stellar mass within inner $10 \mathrm{kpc}\left(M_{\star}^{10}\right)$ and also reproduces the HSC weak lensing signals of massive galaxies with different stellar mass distributions. The best-fitting model shows that halo mass varies significantly at fixed total stellar mass (as much as 0.4 dex) with a clear dependence on $M_{\star}^{10}$. Our two-parameter $M_{\star}^{\text {max }}-M_{\star}^{10}$ description provides a more accurate picture of the galaxy-halo connection at the high-mass end than the simple stellar-halo mass relation (SHMR) and opens a new window to connect the assembly history of haloes with those of central galaxies. The model also predicts that the ex situ component dominates the mass profiles of galaxies at $r<10 \mathrm{kpc}$ for $\log M_{\star} \geq 11.7$. The code used for this paper is available online https://github.com/dr-guangtou/asap
\end{abstract}

Key words: galaxies: elliptical and lenticular, $\mathrm{cD}$-galaxies: formation-galaxies: haloesgalaxies: photometry-galaxies: structure.

\section{INTRODUCTION}

During the last decade, observations and hydrodynamic simulations have significantly furthered our understanding of the formation and assembly of massive galaxies in the nearby Universe. The observed mass assembly (e.g. Lundgren et al. 2014; Ownsworth et al. 2014; Vulcani et al. 2016; also see Bundy et al. 2017) and dramatic structural evolution (e.g. van der Wel et al. 2014; Clauwens et al.

^E-mail: shuang89@ucsc.edu
2017; Hill et al. 2017) support a 'two-phase' formation scenario of massive galaxies (e.g. Oser et al. 2010, 2012; Rodriguez-Gomez et al. 2016). According to this picture, intense dissipation at highredshift swiftly builds up the massive, compact 'core' of today's massive galaxies (e.g. van Dokkum et al. 2008; Damjanov et al. 2009; Toft et al. 2014; van Dokkum et al. 2015; Wellons et al. 2016), including most of the in situ component: stars formed in the main progenitor of the host dark matter halo (e.g. De Lucia \& Blaizot 2007; Genel et al. 2009). Supermassive galaxies, however, are also expected to have a large ex situ component: stars that are accreted from other haloes. After the quenching of star formation 
in massive galaxies, (e.g. Hopkins et al. 2008; Johansson, Naab \& Ostriker 2009; Conroy, van Dokkum \& Kravtsov 2015), the gradual accumulation of the ex situ component dominates the assembly of massive galaxies and helps build-up extended stellar envelopes (e.g. van Dokkum et al. 2008; Bezanson et al. 2009; Huang et al. 2013; Patel et al. 2013). More importantly, these two components should show differences in their spatial distributions as a large fraction of the ex situ component is expected to be deposited at large radii (e.g. Hilz, Naab \& Ostriker 2013; Oogi \& Habe 2013). This suggests that the stellar mass distribution of massive galaxies contains information about their assembly history. From a cosmological perspective, to understand the assembly of massive galaxies is to understand how they hierarchically grow with their dark matter haloes (e.g. Wechsler \& Tinker 2018 and the references within). Recently, the basic understanding of the stellar-halo mass relation (SHMR) has been established using various direct and indirect methods (e.g. Hoekstra 2007; More et al. 2011; Leauthaud et al. 2012a; Behroozi, Wechsler \& Conroy 2013b; Coupon et al. 2015; Zu \& Mandelbaum 2015; van Uitert et al. 2016; Shan et al. 2017; Tinker et al. 2017; Kravtsov, Vikhlinin \& Meshcheryakov 2018). At low redshift, the SHMR can be characterized by a powerlaw relation at low masses, a characteristic pivot halo mass, and an exponential rise at higher masses (Behroozi, Wechsler \& Conroy 2013b; Rodríguez-Puebla et al. 2017; Moster, Naab \& White 2018). Constraints on the SHMR have helped us gain insight into the galaxy-halo connection, but an in-depth picture about how the assembly of galaxies is tied to their dark matter haloes is still lacking. At high-mass end, the situation is particularly true (e.g. Tinker et al. 2017; Kravtsov et al. 2018). First, challenges in measuring the total stellar mass of massive elliptical galaxies with extremely extended light profile (e.g. Bernardi et al. 2013, 2014, 2017; Kravtsov et al. 2018; Pillepich et al. 2018b; Huang et al. 2018c) complicate constraints of the SHMR for massive galaxies. More importantly, this simple scaling relation does not provide the full picture; specifically, it does not describe whether or not the internal structure (i.e. the way in which stellar mass is distributed in massive galaxies) is tied to the assembly history of their dark matter haloes. At high-stellar mass $\left(M_{\star}\right)$ end, the scatter of halo mass at fixed stellar mass is of order 0.3-0.4 dex (e.g. Tinker et al. 2017). In this paper, we seek to explain how similarly massive galaxies can live in haloes with very different mass and assembly histories, by looking for signatures of this assembly process in the stellar mass profiles of massive galaxies. In previous work (Huang et al. 2018a,c, Paper I and Paper II hereafter), we map the stellar mass distributions of massive galaxies at $0.3 \leq z<0.5$ to $>100 \mathrm{kpc}$ individually using deep images from the Hyper SuprimeCam (HSC; Miyazaki et al. 2012) Subaru Strategic Program (SSP, hereafter 'HSC survey'; Aihara et al. 2017a,b). With the help of deep images and the redMaPPer cluster catalogue (e.g. Rozo \& Rykoff 2014; Rykoff et al. 2014), we find evidence that the surface stellar mass density profiles $\left(\mu_{\star}\right)$ of massive central galaxies depend on dark matter halo mass: centrals galaxies in more massive haloes tend to have more extended stellar mass distributions (also see: Charlton et al. 2017; Yoon, Im \& Kim 2017) and less mass in the inner $10 \mathrm{kpc}\left(M_{\star}^{10}\right)$.

Here, we seek to directly confirm this dependence and characterize this relation using the galaxy-galaxy weak lensing (' $\mathrm{g}$ g lensing') method (e.g. Mandelbaum et al. 2006a, b; Leauthaud et al. 2012a; Coupon et al. 2015; Leauthaud et al. 2017) that probes the dark matter halo mass distribution by measuring the coherent shape distortion of background galaxies. Instead of relying on a cluster catalogue, the unprecedented $\mathrm{g}-\mathrm{g}$ lensing capability of the
HSC survey (e.g. Mandelbaum et al. 2018; Medezinski et al. 2018; Miyatake et al. 2018) allows us to map the halo mass trend across a 2D plane described by the $M_{\star}^{10}$ and stellar mass within the largest aperture that is allowed by the depth of the image $\left(M_{\star}^{\max }\right)$ and build an empirical model for galaxy-halo connection at high-mass end.

This paper is organized as follows. We briefly summarize the sample selection and data reduction processes in Section 2. Please refer to Paper I for more technical details. Section 3 describes the weak lensing methodology, and the measurements of aperture $M_{\star}$ and $\mu_{\star}$ profiles are discussed in Section 4. In Section 5, we introduce an empirical model to describe the relation between dark matter halo mass and the distribution of stellar mass within super massive galaxies. The results from our best-fit model are presented in Section 6 and discussed in Section 7. Our summary and conclusions are presented in Section 8.

We use galactic extinction corrected (Schlafly \& Finkbeiner 2011) $A B$ magnitudes (Oke \& Gunn 1983). For cosmology, we assume $H_{0}=70 \mathrm{~km} \mathrm{~s}^{-1} \mathrm{Mpc}^{-1}, \Omega_{\mathrm{m}}=0.3$, and $\Omega_{\Lambda}=0.7$. Stellar mass $\left(M_{\star}\right)$ is derived using a Chabrier initial mass function (IMF; Chabrier 2003). And we use the virial mass for dark matter halo mass $\left(M_{\text {vir }}\right)$ as defined in Bryan \& Norman 1998.

\section{DATA AND SAMPLE SELECTION}

\subsection{SSP S16A data}

In this work, we use the WIDE layer of the internal data release S16A of the HSC SSP, an ambitious imaging survey using the new prime focus camera on the 8.2-m Subaru telescope. These data are reduced by HSCPIPE 4.0 .2 , a specially tailored version of the Large Synoptic Survey Telescope (LSST) pipeline (e.g. Axelrod et al. 2010; Jurić et al. 2015), modified for HSC (Bosch et al. 2017). The coadd images are $\sim 3-4$ mag deeper than SDSS (Sloan Digital Sky Survey; e.g. Abazajian et al. 2009; Aihara et al. 2011; Alam et al. 2015), with a pixel scale of $0^{\prime \prime} 168$. The seeing in the $i$ band has a mean full width at half maximum (FWHM) of 0'.58. Please refer to Aihara et al. (2017a,b) for more details about the survey design and the data products. The general performance of HSCPIPE is validated using a synthetic object pipeline SYNPIPE (e.g. Huang et al. 2018b; code available on github at this link https://github.com/lsst/synpipe). In addition to the full-colour and full-depth cuts, regions that are affected by bright stars are also masked out Coupon et al. (2017). The HSC collaboration compiles the spectroscopic redshifts (spec- $z$ hereafter) of HSC galaxies from a series of available spectroscopic surveys, which is the main source of spec- $z$ in this work. We also include additional spec- $z$ from the most recent data release of the Galaxy And Mass Assembly (GAMA) survey (Driver et al. 2009, 2011; Liske et al. 2015; Baldry et al. 2018) which significantly overlaps with HSC coverage in their G02, G09, G12, and G15 regions and greatly improve the spec- $z$ completeness of our massive galaxy sample. The HSC collaboration also provides photometric redshift (photo- $z$ hereafter) measurements using the point spread function (PSF)-matched fiveband fluxes within 1".5 apertures and six different algorithms. Here, we use the spec- $z$, sample and the photo- $z$ measurements based on the Flexible Regression over Associated Neighbours with Kernel dEnsity estimatioN for Redshifts (FRANKEN-Z; Speagle et al. 2019) algorithm. Please refer to Tanaka et al. (2018) for details about photo- $z$ catalogues.

For our weak lensing measurements, we make use of the first-year shear catalogue described in detail by Mandelbaum et al. (2018). Currently, we use the re-Gaussianization algorithm (Hirata \& Seljak 
2003) to measure galaxy shapes on $i$-band coadd images. Please see Mandelbaum et al. $(2017,2018)$ for more details about our shape measurements and their calibration. Our shape catalogue also excludes a small fraction of the survey area that has a problematic PSF model. The resulting survey area is the full-depth full-colour region for weak lensing analysis (WLFDFC) region, which covers $\sim 137 \mathrm{deg}^{2}$ in all five bands (grizy) to the required imaging depth ( $5 \sigma$ point source detection limit of $26.0 \mathrm{mag}$ ). For our $\mathrm{g}-\mathrm{g}$ lensing measurements, we also use a random catalogue that contains a half million objects and covers the WLFDFC area (e.g. Coupon et al. 2017; Singh et al. 2017).

\subsection{Sample selection}

Our sample selection is very similar to Huang et al. (2018a, c; Paper I and Paper II hereafter). We select all galaxies with $i_{\text {CModel }}<=22.0$ mag and useful five-band cModel photometry in the WLFDFC area. Instead of only using galaxies with spec-z's however, we now assign a best redshift ( $z_{\text {best }}$ ) to each object: We adopt the spec- $z$ when it is available; for others, we use the photo- $z$ measurements from FRANKEN-Z as $z_{\text {best }}$. We select all galaxies within $0.19<$ $z_{\text {best }}<0.51$, where redshift evolution is not a serious concern and the volume is large enough $\left(1.03 \times 10^{8} \mathrm{Mpc}^{3}\right)$ to ensure a large sample of massive galaxies. The performance of FRANKEN$\mathrm{Z}$ at this redshift and magnitude range is unbiased and reliable with respect to the training sample. The typical $1 \sigma$ uncertainty is $\sim 7$ percent with a median bias of about -0.3 percent and typical outlier fraction of 11-19 percent in this redshift range. Compared with the spec-zonly sample, adding in the photo-z's greatly improves the $M_{\star}$ completeness of our sample but does not alter any of our key results. We perform five-band spectral energy distribution (SED) fitting using the CModel photometry to derive the average mass-to-light ratio $\left(M_{\star} / L_{\star}\right)$ of galaxies and initial estimates of $M_{\star}\left(M_{\star}^{\mathrm{cmod}}\right)$. The SED fitting procedure is identical to the one used in Paper I. In short, we use iSEDFit (Moustakas et al. 2013) to measure $M_{\star} / L_{\star}$ ratios and $k$-corrections, assuming the Chabrier (2003) IMF and using the Flexible Stellar Population Synthesis models (FSPS; v2 . 4; Conroy \& Gunn 2010a, b). Please refer to Paper I for more details. Based on the SED fitting results, we select galaxies with $\log _{10}\left(M_{\star, \text { cmodel }} / \mathrm{M}_{\odot}\right)>10.8$ as the initial sample of massive galaxies. Typical uncertainty of $M_{\star}^{\text {cmod }}$ is around $0.05-0.1$ dex. We further measure the $\mu_{\star}$ profiles of these galaxies and aperture $M_{\star}$ within different radii (see Section 4.1).

\section{GALAXY-GALAXY WEAK LENSING METHODOLOGY}

Galaxy-galaxy lensing measures the coherent shape distortion of background galaxies around foreground lens galaxies. Please refer to Mandelbaum et al. (2018) for a detailed description of the construction of our shear catalogue. A detailed description of our method for computing $\Delta \Sigma$ is presented in Speagle et al. 2019). Our methodology is briefly summarized below. The HSC shape catalogue includes a per-galaxy optimal weight defined as

$w_{i}=\frac{1}{e_{\mathrm{rms}}^{2}+\sigma_{e, i}^{2}}$,

where $\sigma_{e, i}$ is the shape measurement error per source galaxy and $e_{\text {rms }}$ is the intrinsic shape noise.

We follow the methodology outlined in Singh et al. (2017) to measure the excess surface mass density (hereafter $\Delta \Sigma$ ) profiles of lens galaxies. Using this method, we measure $\Delta \Sigma$ as:

$\Delta \Sigma_{\mathrm{LR}}(r)=\frac{\Sigma_{\mathrm{Ls}} w_{\mathrm{Ls}} \gamma_{t}^{(\mathrm{ls})} \Sigma_{\mathrm{crit}}^{(\mathrm{Ls})}}{\Sigma_{\mathrm{Ls}} w_{\mathrm{Ls}}}-\frac{\Sigma_{\mathrm{Rs}} w_{\mathrm{Rs}} \gamma_{t}^{(\mathrm{Rs})} \Sigma_{\mathrm{crit}}^{(\mathrm{Rs})}}{\Sigma_{\mathrm{Rs}} w_{\mathrm{Rs}}}$,

where we use $L$ for a real-lens galaxy and $R$ for random point. The superscript or subscript Ls indicates measurement for lens-source pair, while Rs means the measurement for random-source pair. $\gamma$ is the tangential shear, $w$ is the weight, and $\Sigma_{\text {crit }}$ is the critical surface density defined as:

$\Sigma_{\text {crit }}=\frac{c^{2}}{4 \pi G} \frac{D_{\mathrm{A}}\left(z_{\mathrm{s}}\right)}{D_{\mathrm{A}}\left(z_{1}\right) D_{\mathrm{A}}\left(z_{1}, z_{\mathrm{s}}\right)\left(1+z_{1}\right)^{2}}$,

where $D_{\mathrm{A}}\left(z_{\mathrm{L}}\right), D_{\mathrm{A}}\left(z_{\mathrm{s}}\right)$, and $D_{\mathrm{A}} z_{\mathrm{L}}, z_{\mathrm{s}}$ are the angular diameter distances to lens (random), source, and between them, respectively. We use 11 radial bins uniformly spaced in log-space from $200 \mathrm{kpc}$ to $10 \mathrm{Mpc}$ (physical units are assumed). The redshift distribution of random points is matched to the lens sample. The subtraction of signal around random positions helps remove overestimated jackknife errors (e.g. Clampitt et al. 2017; Shirasaki et al. 2017) and accounts for non-negligible coherent additive bias of the shear measurements (e.g. Takada \& Hu 2013). This method has been adopted by the Dark Energy Survey (DES; e.g. Prat et al. 2017) and the Kilo-Degree Survey (KiDS; e.g. Amon et al. 2018). We selected source galaxies based on the following criteria. First, a set of photo- $z$ quality cuts are applied to the sample; these are the basic cuts that are described in Speagle et al. 2019). For each lens, we further require $z_{\mathrm{s}}-z_{\mathrm{L}} \geq 0.1$ and $z_{\mathrm{s}}>z_{\mathrm{L}}+\sigma_{\mathrm{s}, 68}$, where $\sigma_{\mathrm{s}, 68}$ is the $1 \sigma$ confidence interval of the source photo- $z$. Errors are estimated via jackknife resampling. We divide the S16A WLFDFC footprint into 41 roughly equal-area jackknife regions with regular shapes. In practice, the effective number of jackknife regions varies, depending on the specific subsample of lenses. Typically $N_{\mathrm{JK}}>30$. The diagonal errors for $\Delta \Sigma$ are then estimated as:

$\operatorname{Var}_{\mathrm{Jk}}(\widehat{\Delta \Sigma})=\frac{N_{\mathrm{Jk}}-1}{N_{\mathrm{Jk}}} \sum_{i=1}^{N_{\mathrm{Jk}}}\left(\Delta \Sigma_{i}-\overline{\Delta \Sigma}\right)^{2}$,

where $N_{\mathrm{Jk}}$ is the number of jackknife regions, $\Delta \Sigma_{i}$ is the $\Delta \Sigma$ profile in each region, and $\overline{\Delta \Sigma}$ is the mean profile among all jackknife regions.

We measure the stacked $\Delta \Sigma$ profiles of massive galaxies using a pure Python $\mathrm{g}-\mathrm{g}$ lensing pipeline designed for the HSC survey: dsigma (available here: https://github.com/dr-guangtou/dsigma). Please refer to Speagle et al. 2019) for more technical details of dsigma and the g-g lensing measurements.

\section{MEASUREMENTS}

\section{1 $\mu_{\star}$ profiles and aperture stellar masses}

We measure 1D surface brightness profiles on the HSC $i$-band images which typically have the best imaging conditions. We use the Ellipse task from IRAF package after fixing the shape of the isophote and adaptively masking out all neighbouring objects based on their brightness and distance to the target. The 1D surface brightness profile is based on the median flux of unmasked pixels along each isophote after $3 \sigma$-clipping the pixels twice. ${ }^{1}$ Since we limit the sample at $z>0.2$, the angular sizes of these galaxies make

\footnotetext{
${ }^{1}$ We use projected 2D stellar mass maps from hydrosimulation to show that our profiles are robust against the impact of unmasked flux from other objects (Ardilla et al. in preparation).
} 
them less affected by the oversubtraction caused by the HSCPIPE used in S16A reduction. ${ }^{2}$ We also model the background of the cutout image after aggressively masked out all pixels with detections. We use this empirical sky model to correct the background and make sure the flux distribution of sky pixels is centred at 0.0 . These 1D profiles are robust within inner 100-150 kpc against different systematics. Using the average $M_{\star} / L_{\star}$ measured from SED fitting, we then convert these profiles into surface density profiles of stellar mass - denoted $\mu_{\star}$. Integration of the $\mu_{\star}$ profiles provides us with $M_{\star}$ within an elliptical aperture. Paper I contains more technical details about our procedure. We can reliably derive $\mu_{\star}$ profiles out to more than $100 \mathrm{kpc}$ for individual massive galaxies without being limited by the background subtraction. On small scales, our profiles are resolved down to $\sim 5-6 \mathrm{kpc} .^{3}$ In Paper I and Paper II, we use $M_{\star}$ within $10 \mathrm{kpc}\left(M_{\star}^{10}\right)$ and $100 \mathrm{kpc}\left(M_{\star}^{100}\right)$ as measures of the inner and 'total' $M_{\star}$ of a galaxy. We also show that $M_{\star}^{10}$ can be used as a rough proxy for the mass of the in situ component. In this work, instead of continuing to use $M_{\star}^{100}$, we choose to use the maximum 1D stellar mass $\left(M_{\star}^{\max }\right)$ to compare with the total stellar mass predicted by our model. This choice integrates the $\mu_{\star}$ profile to the radius where the median intensity is consistent with the standard deviation of the sky background. We have shown that $M_{\star}^{\text {max }}$ on average adds another 0.03-0.05 dex of $M_{\star}$ compared with $M_{\star}^{\star 100}$; hence, this approach should bring us a little closer to the true 'total' $M_{\star}$. This choice is motivated by the assumption of the empirical model but does not change the key results of this work, which we explain in Section 5. As was the case in Paper I, we cannot derive $1 \mathrm{D}$ profiles for $\sim 11$ per cent of massive galaxies due to strong contamination (e.g. a bright star or foreground galaxy) or complex inner structure (e.g. on-going major merger).$^{4}$ Meanwhile, as shown in Huang et al. (2018b), HSCPIPE tends to classify some stars as extended objects. We find that these contaminations can be easily picked up as outliers on the $M_{\star}^{100}-M_{\star}^{10}$ plane and removed using $\log \left(M_{\star, \text { tot }} / \mathrm{M}_{\odot}\right)-\log _{10}\left(M_{\star, 10 \mathrm{kpc}} / \mathrm{M}_{\odot}\right) \leq 0.03$. In this work we ignore the $M_{\star} / L_{\star}$ gradients. Based on Roediger \& Courteau (2015), ${ }^{5}$ a colour difference of $\Delta(g-i)=0.2$, which is roughly the average $g-i$ colour difference between 10 and $100 \mathrm{kpc}$, translates into an $M_{\star} / L_{\star}$ difference of $\Delta \log \left(M_{\star} / L_{i}\right) \sim 0.15$. Considering that the cModel photometry measures the average colour for the main body of massive galaxies, we believe that the systematic uncertainty caused by ignoring the colour gradient should smaller than this value. Assuming a negative colour gradient, we may be slightly underestimating $M_{\star}^{10}$ while slightly overestimating $M_{\star}^{\max }$. We also want to point out that the 1D $M_{\star}^{\text {max }}$ still likely to miss a fraction of $M_{\star}$ due to the limited imaging depth and background uncertainty. Using similarly massive galaxies from the IllustrisTNG simulation, we find that the missing $M_{\star}$ outside $100 \mathrm{kpc}$ is typically smaller than 0.1 dex and show weak but positive correlation with stellar mass (Ardila et al. in preparation). If this is indeed the case for real massive galaxies, the key results here will not be affected. Meanwhile, Sérsic model fitting or stacking analysis of these massive galaxies sometime reveal a significant amount of missing $M_{\star}$ outside $100 \mathrm{kpc}$ (e.g. Sonnenfeld, Wang \& Bahcall 2019; Zhang

\footnotetext{
${ }^{2}$ The 'superpixel' size for sky measurement is $128 \times 128$ pixels whose half-size corresponds to $\sim 210 \mathrm{kpc}$ at $z=0.2$

${ }^{3} 1.0$ arcsec corresponds to 3.2 and $6.17 \mathrm{kpc}$ at $z=0.19$ and 0.51 , respectively; while the mean $i$ band seeing has FWHM $=0$ '.58.

${ }^{4}$ The $M_{\star}^{\text {cmod }}$ distribution of these galaxies is similar to the whole sample; hence, excluding them should not bias our model.

${ }^{5} \log \left(M_{\star} / L_{i}\right)=0.83 \times(g-i)-0.597$ for the FSPS stellar population model.
}

et al. 2019) although this depends on the validity of the model assumption and the reliability of the stacking technique. We will further look into this issue in future works. Our sample contains 38653 galaxies with $\log _{10}\left(M_{\star, \max } / \mathrm{M}_{\odot}\right) \geq 11.0$ at $0.19 \leq z \leq 0.51$. 57 per cent of them have spec- $z$ 's.

\subsection{Stellar mass functions}

In this work, we estimate the stellar mass function (SMF) of $M_{\star}^{\max }$ $\left(\Phi_{\max }\right)$ in seven bins between $11.6 \leq \log _{10}\left(M_{\star, \max } / \mathrm{M}_{\odot}\right)<12.3$, while we estimate the SMF of $M_{\star}^{10}\left(\Phi_{10}\right)$ in ten bins between 10.8 $\leq \log _{10}\left(M_{\star, 10 \mathrm{kpc}} / \mathrm{M}_{\odot}\right)<11.8$. We separate the current WLFDFC area into 30 smaller regions, and derive uncertainties via jackknife resampling. We add a 10 percent uncertainty to represent the potential impact of galaxies without a useful 1D profile. We take the uncertainty of $M_{\star}$ measurements into account by integrating the normalized posterior distribution function (PDF) of the $M_{\star}$ of each galaxy $^{6}$ to estimate its contribution in each $M_{\star}$ bin. For a given $M_{\star}$ bin with lower and upper boundary of $M_{1}$ and $M_{\mathrm{u}}$, the effective number of galaxies in the bin is:

$N_{\text {eff }}=\sum_{i=1}^{n_{\text {gal }}} \frac{1}{2}\left[\operatorname{erf}\left(\frac{M_{\mathrm{u}}-M_{i}}{\sqrt{2} \sigma_{i}}\right)-\operatorname{erf}\left(\frac{M_{1}-M_{i}}{\sqrt{2} \sigma_{i}}\right)\right]$,

where $M_{i}$ is the mean $M_{\star}$ and $\sigma_{i}$ is the uncertainty for each massive galaxy and erf() is the error function. By comparing our results with SMFs from the PRIsm MUlti-object Survey (PRIMUS; e.g. Moustakas et al. 2013) at a similar redshift, we find that massive galaxies with $\log _{10}\left(M_{\star, \max } / \mathrm{M}_{\odot}\right) \geq 11.6$ are a mass complete sample and are considered in the following modelling. In total, we have 6481 and 3156 galaxies at $\log _{10}\left(M_{\star, \max } / \mathrm{M}_{\odot}\right) \geq 11.5$ and $\geq 11.6$; 5756 and 2944 of them have spec- $z$. The $M_{\star}^{\text {max }}-M_{\star}^{10}$ distribution of our sample is shown in Figs 1 and 4. The SMFs of $M_{\star}^{\max }$ and $M_{\star}^{10}$ for the $\log _{10}\left(M_{\star, \max } / \mathrm{M}_{\odot}\right) \geq 11.6$ sample are shown in panel (b) of Fig. 4. The SMFs of $M_{\star}^{\max }$ and $M_{\star}^{10}$ are highly correlated as $M_{\star}^{10}$ is included in the measurement of $M_{\star}^{\max }$. We calculate the covariance matrix of the joint $M_{\star}^{\text {max }}-M_{\star}^{10}$ SMF using the same jackknife samples.

\subsection{Galaxy-galaxy lensing signals across the aperture mass plane}

In Paper II (see Fig. 3), we find that massive central galaxies of redMa PPer clusters (e.g. Rozo \& Rykoff 2014; Rykoff et al. 2014) have lower $M_{\star}^{10}$ than those in less massive haloes at fixed $M_{\star}^{100}$, which suggests that the stellar mass distributions in massive central galaxies depend on their $M_{\text {vir }}$. Therefore, we expect a gradient of $M_{\mathrm{vir}}$ across the aperture mass plane. Our goal is to map out this gradient directly using weak lensing and without relying on any redMaPPer cluster catalogue. Panel (a) in Fig. 1 shows the distribution of massive galaxies over the $M_{\star}^{\max }-M_{\star}^{10}$ plane. We group galaxies into three sub-samples based on the ranking of their $M_{\star}^{10}$ at fixed $M_{\star}^{\max }$, following a similar strategy employed in Mao, Zentner \& Wechsler (2018). As illustrated in the inset panel, the three sub-samples share almost identical distributions of $M_{\star}^{\max }$. Therefore, they represent massive galaxies with different stellar mass distributions at the same 'total' stellar mass, as proved by their median $\mu_{\star}$ profiles (panel b of Fig. 1). Galaxies with lower $M_{\star}^{10}$ have lower $\mu_{\star}$ on small radial scales and have larger extended

\footnotetext{
${ }^{6} \mathrm{We}$ assume that the PDF is described by a Gaussian distribution.
} 

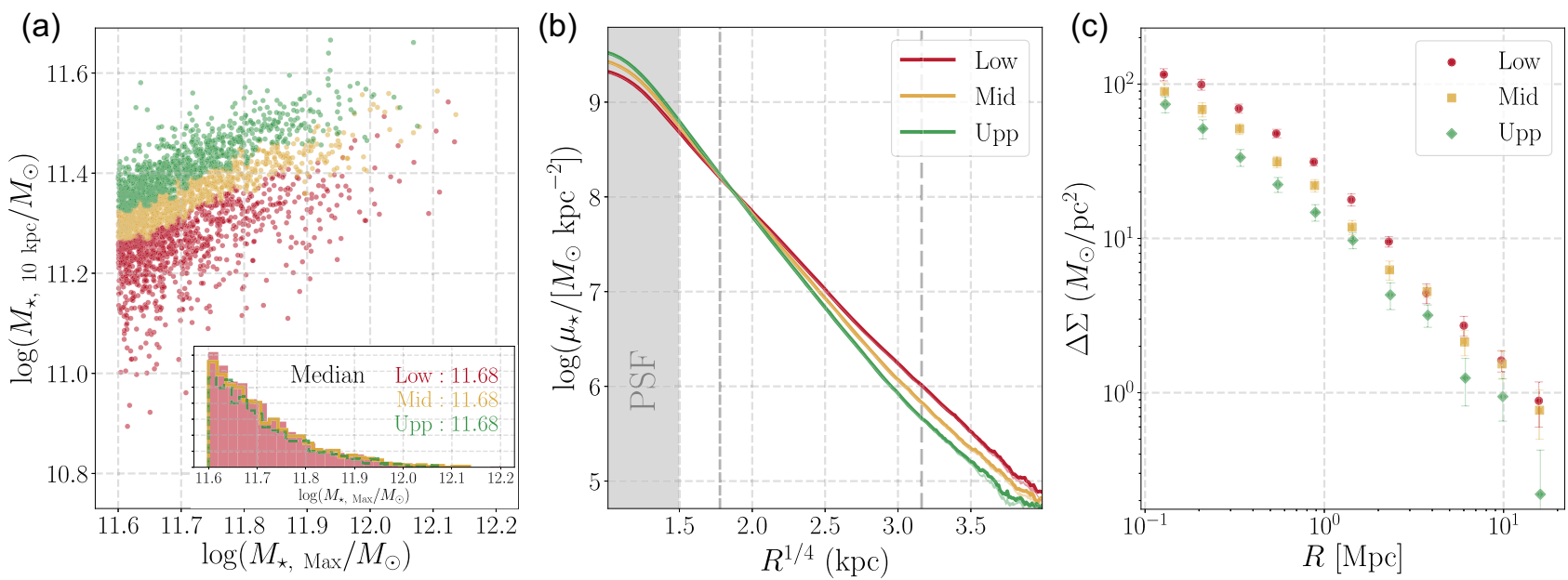

Figure 1. (a) Distribution of massive galaxies on the aperture mass plane. We group massive galaxies into three subsamples based on the ranking of their $M_{\star}^{10}$ at fixed $M_{\star}^{\max }$. The inset plot demonstrates that the three subsamples share similar distributions of $M_{\star}^{\max }$. (b) Median surface mass density profiles of the subsamples visualize the differences in their stellar mass distributions. The $x$-axis employs an $R^{1 / 4}$ scaling. The shaded region within $\sim 5 \mathrm{kpc}$ highlights the region affected by seeing. Two dashed lines label the $10 \mathrm{kpc}$ (short) and $100 \mathrm{kpc}$ (long) radius. (c) The stacked g-g lensing signals prove that at fixed $M_{\star}^{\max }$, massive galaxies with lower $M_{\star}^{10}$ tend to live in more massive dark matter haloes. The Jupyter notebook for this figure is available here: https://github.com/dr-guangtou/asap/blob/master/note/fig1.ipynb.

outer envelopes. The median $\mu_{\star}$ profiles cross each other at $\sim 12-$ $15 \mathrm{kpc}$, close to the effective radius $\left(R_{\mathrm{e}}\right)$ of these galaxies. We then measure the stacked $\Delta \Sigma$ profiles of these three sub-samples using the method described in Section 3. The results are displayed in panel (c) of Fig. 1. It is very clear from this figure that, on average, massive galaxies with lower $M_{\star}^{10}$ have higher $\Delta \Sigma$ signals indicating that they live in more massive dark matter haloes. This confirms the expected trend across the aperture mass plane that was first identified in Paper II using broad $M_{\text {vir }}$ bins from cluster catalogue. Thanks to the impressive weak lensing capabilities of the HSC survey, we can further group massive galaxies into bins of $M_{\star}^{\max }$ and $M_{\star}^{10}$ and investigate the variation of their stacked $\Delta \Sigma$ profiles and halo masses. The Jupyter notebook for measuring these $\Delta \Sigma$ profiles can be found here: https://github.com/dr-guangtou/asap/blob/maste $\mathrm{r} /$ note/hsc_weak_lensing.ipynb. We also make a GIF animation to visualize this variation: https://github.com/dr-guangtou/asap/blob /master/doc/dsig_over_aperture_plane.gif. To account for scatter in $M_{\text {vir }}$ within each $M_{\star}^{\max }-M_{\star}^{10}$ 'box', the impact of satellites, and the two-halo term, we model our lensing signals using a full forward model based on $N$-body simulations and a state-of-the-art semiempirical model. We will group our massive galaxies into 12 bins of aperture masses while making sure that (1) there are enough massive galaxies in each bin so that the stacked $\Delta \Sigma$ profile has good S/N; and (2) the $M_{\star}^{10}$ bins at fixed $M_{\star}^{\max }$ represent massive galaxies with different stellar mass in the inner region. We explain the details of the model in Section 5.

\section{MODELLING THE $M_{\mathrm{VIR}}-M_{\star}^{\max }-M_{\star}^{10}$ RELATION}

\subsection{Goals of the Model}

Our goal is to construct a model that connects the hierarchical growth of dark matter haloes to the assembly and structure of high-mass central galaxies. Ideally, we could directly compare with predictions from cosmological hydrodynamical simulation, such as Illustris (e.g. Genel et al. 2014; Vogelsberger et al.
2014) or EAGLE (e.g. Crain et al. 2015; Schaye et al. 2015) that are being used to study the evolution of massive galaxies (e.g. Rodriguez-Gomez et al. 2016; Wellons et al. 2016; Qu et al. 2017); or with the high-resolution zoom-in hydrosimulation of galaxy groups or clusters, such as Rhapsody-G (e.g. Hahn et al. 2017), Hydrangea (e.g. Bahé et al. 2017), and FABLE (e.g. Henden et al. 2018) simulations, which can better capture the impact of important physical process like the AGN feedback. However, current hydrosimulations typically lack of the volume or sample size to statistically study galaxies at the high- $M_{\text {vir }}$ end. In addition, we also want a model with the flexibility (free parameters) to fit the actual observations. An alternative approach would be to use a semi-analytic model (SAM) based on dark matter simulations and approximate physical recipes (e.g. White \& Frenk 1991; Benson \& Bower 2010; Guo et al. 2011; Henriques et al. 2015; Somerville, Popping \& Trager 2015; Croton et al. 2016) could be another approach. However, while recent progress has been made in this area, fitting the large numbers of parameters that a SAM typically uses is still non-trivial (e.g. Lu et al. 2011; Benson 2014, 2017). For these reasons, we base our formalism on the recently developed semi-empirical model approach (e.g. Becker 2015; RodríguezPuebla et al. 2017; Behroozi et al. 2019; Moster et al. 2018). This new methodology makes rather minimal a priori assumptions about the galaxy-halo connection, and is constrained by observations at different redshifts (stellar mass growth, star-formation history, and clustering properties of galaxies across a wide range of halo masses and redshifts). This results in a model that can predict the properties of individual galaxies and how they connect with the full assembly history of their dark matter haloes.

\subsection{Simulations and UniverseMachine framework}

UniverseMachine (Behroozi et al. 2019; code available here:ht tps://bitbucket.org/pbehroozi/universemachine) is a massively parallel implementation of a semi-empirical modelling method. It is capable of reproducing key observations (e.g. SMFs, star formation 
rates, and quenched fractions) over a large range of stellar masses and redshifts. For a given halo from a cosmological simulation, UniverseMachine parametrizes its star formation rate (SFR) as a function of halo mass, halo accretion rate, and redshift. UniverseMachine exploits the Markov Chain Monte Carlo (MCMC) Bayesian method to compare results with a series of compiled observations. The UniverseMachine model we use here is based on the Small MultiDark Planck (SMDPL) simulation, which is part of the MultiDark simulation series using a Planck cosmology. It has a $400 \mathrm{Mpc}^{-1}$ simulation box size and uses $3840^{3}$ particles. The dark matter mass resolution is $10^{8} \mathrm{M}_{\odot} h^{-1}$. The volume of the SMDPL simulation is two times larger than the volume from which our HSC sample at $0.19 \leq z \leq 0.51$ is drawn from. Dark matter subhalo properties are extracted using the Rockstar (Behroozi, Wechsler \& Wu 2013a) halo finder with merger trees generated by the Consistent Trees code. Halo mass is defined as the mass within the virial radius $\left(M_{\mathrm{vir}}\right)$ using the formula from Bryan \& Norman (1998). For satellite galaxies, we will also use their peak $M_{\text {vir }}$ over their accretion history $\left(M_{\text {peak }}\right)$. Here we use the snapshot at $z \sim 0.37$, which is very close to the mean redshift of our sample $(\bar{z} \sim 0.32)$. The fiducial UniverseMachine model predicts a 'galaxy mass' and an 'ICL' mass. During mergers, a fraction of stars from the incoming satellite become unbound by the gravitational well of the galaxy and are added to the 'ICL' component. Although there is evidence for an unbound diffuse stellar component around nearby massive galaxies (e.g. Kelson et al. 2002; Bender et al. 2015; Longobardi et al. 2015), the main motivation of this approach is to make sure the SMF matches observational constraints at low redshift, otherwise UniverseMachine overproduce the SMF at the high- $M_{\star}$ end (Behroozi et al. 2019). However, as we showed in Paper I, it is extremely difficult to photometrically separate out a physically meaningful 'ICL' component. More importantly, the ICL component is also an integrated part of the assembly history of massive galaxies and should be taken into account when studying their galaxy-halo connection. Therefore, instead of using the 'galaxy' and 'ICL' separation, we use a specially tailored UniverseMachine model that provides a more physically motivated decomposition of stars in massive galaxies: for each galaxy, our UniverseMachine model will predict the mass of the in situ and ex situ components $\left(M_{\star}^{\text {ins }}\right.$ and $\left.M_{\star}^{\text {exs }}\right)$. As mentioned earlier, these are stars formed inside and outside the main progenitor of the sub-halo. For each galaxy, the stellar mass of the galaxy $\left(M_{\star}^{\text {gal }}\right)$ is simply the sum of $M_{\star}^{\text {ins }}$ and $M_{\star}^{\text {exs }}$. The stellar mass of the central galaxy in a halo is denoted as $M_{\star}^{\text {cen }}$. For each halo, we also calculate the total stellar mass within the halo $\left(M_{\star}^{\text {all }}\right)$ meaning the sum of stellar mass of the central and all satellites. These stellar mass definitions are given in Table 1.

\subsection{ASAP model}

In this section, we explain the design and key assumptions behind our empirical model, which we call the $\operatorname{ASAP}^{7}$ model. Constrained by the observed SMFs of different aperture masses and $\Delta \Sigma$ profiles across the aperture mass plane, the ASAP model will connect $M_{\mathrm{vir}}$, $M_{\star}^{\text {ins }}$, and $M_{\star}^{\text {exs }}$ to the observed stellar mass distributions among massive galaxies. The ASAP model is based on the following two key ingredients:

\footnotetext{
${ }^{7}$ An initialism for Alexie Leauthaud, Song Huang, Andrew Hearin, and Peter Behroozi, the first names of the main contributors.
}

(i) There is a tight log-log linear relation between halo mass and the total stellar mass within the halo (TSHMR) at the high- $M_{\text {vir }}$ end (Bradshaw et al. 2019).

(ii) The UniverseMachine model provides in situ and ex situ components - we add a prescription to describe the spatial distributions of these components.

\subsubsection{Total stellar-halo mass relation (TSHMR)}

The SHMR is the relation between halo mass and central galaxy mass. Whereas the slope of the SHMR varies with $M_{\text {vir }}$ and the scatter of $M_{\mathrm{vir}}$ at fixed $M_{\star}$ is large at the high- $M_{\star}$ end, recent hydrosimulations (e.g. Pillepich et al. 2018a) and semi-empirical models (e.g. Behroozi et al. 2019; Bradshaw et al. 2019) suggest that the TSHMR follows the simple tight, log-linear correlation with $M_{\text {vir }}$ (at least at the high- $M_{\text {vir }}$ end). ${ }^{8}$ Motivated by this, we place the TSHMR at the core of our approach. The SHMR then emerges as a consequence of the TSHMR and the assembly histories of haloes (e.g. Bradshaw et al. 2019). We assume a log-linear relation between the mass of the host dark matter halo $\left(M_{\mathrm{vir}}\right)$ and the total stellar mass within the halo (including the central galaxy, satellites from all sub-haloes, and the ICL component; $\left.\mathcal{M}_{\star}^{\text {all }}\right)$. The TSHMR used in the ASAP model is described as:

$\log \mathcal{M}_{\star}^{\text {all }}=a \times\left(\log M_{\mathrm{vir}}-13.5\right)+b$.

The slope $(a)$ and intercept $(b)$ are free parameters in our model. We adopt a pivot $M_{\mathrm{vir}}$ of $\log _{10}\left(M_{\mathrm{vir}} / \mathrm{M}_{\odot}\right)=13.5$ in all $\log$-linear scaling relations involving halo mass to reduce the degeneracy between the slope and intercept. The exact value of this pivot mass does not impact our results. The scatter in this relation is also modelled as a simple log-linear relation:

$\sigma_{\log \mathcal{M}_{\star}^{\text {all }}}=c \times\left(\log M_{\mathrm{vir}}-13.5\right)+d$,

where $c$ and $d$ are two additional parameters. Here $d$ is the $\sigma_{\log } \mathcal{M}_{\star}^{\text {all }}$ at $\log M_{\text {vir }}=13.5$. We also choose a hard-coded lower limit of scatter at $\sigma_{\log \mathcal{M}_{\star}^{\text {all }}}=0.01$ to avoid the non-physical negative scatter value made possible by the priors of $c$ and $d$. We will discuss this choice further in the following section. The above relations determine the total amount of $M_{\star}$ in each 'parent' halo in the ASAP model. We should point out that, when comparing to observations, the scatter should be a combination of the intrinsic scatter of the TSHMR and the measurement errors of observed stellar mass and weak lensing profiles. We will discuss the scatter of TSHMR further in Section 6.3.1. So far, our model has simply 'pasted' $\mathcal{M}_{\star}^{\text {all }}$ values on haloes in our simulations. The information that we adopt from the UniverseMachine is the following. The UniverseMachine model tells us, for a given $\mathcal{M}_{\star}^{\text {all }}$, how mass is divided up among galaxies. For every galaxy, we compute $\delta_{\text {gal }} \equiv \mathcal{M}_{\star}^{\text {gal }} / \mathcal{M}_{\star}^{\text {all }}$. At this stage, we also forward model uncertainties associated with stellar mass measurements Thus, each galaxy in our mock catalogue is assigned a mass following:

$\log \mathcal{M}_{\star, \text { gal }} \sim \mathcal{N}\left(\log \left(\mathcal{M}_{\star}^{\text {all }} \times \delta_{\text {gal }}\right), \sigma_{\left.\log \mathcal{M}_{\star}^{\text {all }}\right),}\right.$

where $\mathcal{N}(\mu, \sigma)$ is a normal distribution with mean value of $\mu$ and standard deviation of $\sigma$. We apply this model to both

${ }^{8}$ In observations, the total Ks-band luminosity or stellar mass in galaxy groups and clusters also show tight, $\log$-linear relation with halo mass (e.g. Lin \& Mohr 2004; Leauthaud et al. 2012b; Budzynski et al. 2014; van der Burg et al. 2014; Patel et al. 2015; Ziparo et al. 2016; Kravtsov et al. 2018). 
Table 1. Definitions of halo masses and stellar masses used in this work. Rows with different colours are used to separate the masses defined in observations (blue), in (SMDPL) dark matter simulation (orange), in the UniverseMachine model predictions (green), and in the Accelerated SAP (ASAP) model developed in this work (red). Notation with calligraphic letters is used to denote the ASAP model predictions.

\begin{tabular}{|c|c|c|}
\hline Symbol & Origin & Explanation \\
\hline $\begin{array}{c}M_{\star}^{10} \\
M_{\star}^{\max }\end{array}$ & $\begin{array}{l}\text { Observation } \\
\text { Observation }\end{array}$ & $\begin{array}{c}\text { Aperture } M_{\star} \text { within inner } 10 \mathrm{kpc} \text { via integrating the } 1-\mathrm{D} \mu_{\star} \text { profile } \\
\text { Integrated } M_{\star} \text { with the maximum radius of the measured } 1-\mathrm{D} \mu_{\star} \text { profile }\end{array}$ \\
\hline $\begin{array}{c}M_{\text {vir }} \\
M_{\text {peak }}\end{array}$ & $\begin{array}{l}\text { SMDPL } \\
\text { SMDPL }\end{array}$ & $\begin{array}{c}\text { Dark matter halo mass within virial radius (Bryan \& Norman 1998) } \\
\text { Peak historical dark matter halo mass }\end{array}$ \\
\hline$M_{\star}^{\text {all }}$ & UniverseMachine & $M_{\star}$ of all galaxies (central and satellites) within a host dark matter halo \\
\hline$M_{\star}^{\text {gal }}$ & UniverseMachine & $M_{\star}$ of a central or satellite galaxy \\
\hline$M_{\star}^{\text {cen }}$ & UniverseMachine & $M_{\star}$ of the central galaxy \\
\hline$M_{\star}^{\text {ins }}$ & UniverseMachine & $M_{\star}$ of the in situ component of a galaxy \\
\hline$M_{\star}^{\text {exs }}$ & UniverseMachine & $M_{\star}$ of the ex situ component of a galaxy \\
\hline$\delta_{\text {gal }}$ & UniverseMachine & $M_{\star}^{\text {gal }} / M_{\star}^{\text {all }}:$ stellar mass fraction of galaxy in the halo \\
\hline$\delta_{\text {ins }}$ & UniverseMachine & $M_{\star}^{\text {ins }} / M_{\star}^{\text {gal }}$ : stellar mass fraction in situ component in the galaxy \\
\hline$\delta_{\text {exs }}$ & UniverseMachine & $M_{\star}^{\text {exs }} / M_{\star}^{\text {gal }}$ : stellar mass fraction ex situ component in the galaxy \\
\hline$M_{\star}^{\text {all }}$ & ASAP & Model predicted total $M_{\star}$ in a host dark matter halo \\
\hline $\mathcal{M}_{\star}^{\text {gal }}$ & ASAP & Model predicted $M_{\star}$ of a galaxy (central or satellite) \\
\hline $\mathcal{M}_{\star}^{\text {cen }}$ & ASAP & Model predicted $M_{\star}$ of a central galaxy \\
\hline $\mathcal{M}_{\star}^{\mathrm{ins}}$ & ASAP & Model predicted in situ $M_{\star}$ \\
\hline $\mathcal{M}_{\star}^{\text {exs }}$ & ASAP & Model predicted ex situ $M_{\star}$ \\
\hline $\mathcal{M}_{\star}^{\mathrm{in}, 10}$ & ASAP & Model predicted in situ $M_{\star}$ within inner $10 \mathrm{kpc}$ \\
\hline $\mathcal{M}_{\star}^{\mathrm{ex}, 10}$ & ASAP & Model predicted ex situ $M_{\star}$ within inner $10 \mathrm{kpc}$ \\
\hline $\mathcal{M}_{\star}^{10}$ & ASAP & Model predicted $10 \mathrm{kpc}$ aperture $M_{\star}$ \\
\hline
\end{tabular}

centrals and satellite galaxies. Massive satellites are included in our forward modelling process because we do not attempt to distinguish centrals and satellites in our HSC sample. ${ }^{9}$ The reason we choose this approach instead of directly comparing with the UniverseMachine model predictions (e.g. $\mathcal{M}_{\star}^{\text {all }}$ or $\mathcal{M}_{\star}^{\text {cen }}$ ) is that the current UniverseMachine model does not reproduce the HSC SMFs for massive galaxies well. The UniverseMachine 'galaxy' underestimates the SMF at high- $M_{\star}$ end when compared to HSC since it is tuned to match the SMFs from Muzzin et al. (2013) that contain larger numbers of massive galaxies but do not capture the light in the outskirt of low- $z$ massive galaxies (see Behroozi et al. 2019). However, if we combine the 'galaxy' and the 'intrahalo light' (IHL) components, the total mass significantly overpredicts the HSC SMFs instead. Therefore, we assume that the halo and stellar mass assembly in the UniverseMachine model can still correctly capture the relative mass contribution of each galaxy in the halo. In the near future, we will try to incorporate

${ }^{9}$ Uncertainties of photometric redshifts make it difficult to accurately separate centrals and satellites. Meanwhile, the satellite fraction at $\log _{10}\left(M_{\star, \max } / \mathrm{M}_{\odot}\right) \geq 11.5$ is less than $<10$ percent; see Sallaberry et al. (in preparation). the HSC SMFs into the observational constraints of Universe Machine.

\subsubsection{Spatial distributions of in situ and ex situ stars}

For every galaxy, the second ingredient that we inherit from the UniverseMachine is the fraction of in situ and ex situ component $\left(\delta_{\text {ins }}\right.$ and $\delta_{\text {exs }}$ ). We now model the observed aperture masses, $M_{\star}^{10}$ and $M_{\star}^{\text {max }}$, via a prescription that describes the spatial distributions of in situ and ex situ stars. First, we assume the observed $M_{\star}^{\max }$ is a good proxy for the 'total' stellar mass of the galaxy:

$\mathcal{M}_{\star}^{\max }=\mathcal{M}_{\star}^{\mathrm{ins}}+\mathcal{M}_{\star}^{\mathrm{exs}}$.

Next, we predict $M_{\star}^{10}$ using two assumptions. First, we assume that a fixed fraction of the in situ component is within the inner $10 \mathrm{kpc}$ of the galaxy:

$\mathcal{M}_{\star}^{\text {in, } 10}=f_{\text {ins }} \times \mathcal{M}_{\star}^{\text {ins }}$.

Secondly, we assume that the fraction of ex situ stars within $10 \mathrm{kpc}$ depends on halo mass:

$\mathcal{M}_{\star}^{\mathrm{ex}, 10}=f_{\mathrm{exs}} \times \mathcal{M}_{\star}^{\mathrm{exs}}$, 
where the relation between the fraction and halo mass is described by:

$f_{\mathrm{exs}}=A_{\mathrm{exs}} \times\left(\log M_{\mathrm{vir}}-13.5\right)+B_{\mathrm{exs}}$.

Given these two assumptions, the predicted $10 \mathrm{kpc}$ aperture mass is then: $\mathcal{M}_{\star}^{10}=\mathcal{M}_{\star}^{\text {in,10 }}+\mathcal{M}_{\star}^{\text {ex,10}}$. To model $M_{\star}^{10}$ therefore requires three extra free parameters: $f_{\text {ins }}, A_{\text {exs }}$, and $B_{\text {exs }}$. These assumptions are made to ensure a simple model with a small number of free parameters. Meanwhile, they are also partially motivated by the relationships for massive galaxies in the IllustrisTNG simulation (Chowdhury et al. in preparation).

For satellite galaxies, we use $M_{\text {peak }}$ instead of $M_{\text {vir }}$. However, because the fraction of satellite galaxies that are massive enough to be included in our sample is very low at the high-stellar mass end (Bradshaw et al. in preparation), this choice has no impact on our results. As the scatter of the TSHMR is designed to carry both intrinsic scatter and measurement uncertainties of stellar mass, the predicted $\mathcal{M}_{\star}^{10}$ and $\mathcal{M}_{\star}^{\mathrm{Max}}$ will be described by normal distributions with the same scatter.

In total, our model has seven free parameters: two for the TSHMR; two for the scatter of the TSHMR; and three for the fraction of in situ and ex situ stars within $10 \mathrm{kpc}$. Fig. 2 is a visualization of our model.

\subsubsection{Predictions for the $S M F$ s and $\Delta \Sigma$ profiles}

We predict the SMFs of $\mathcal{M}_{\star}^{10}$ and $\mathcal{M}_{\star}^{\text {Max }}$ using the same method and in the same stellar mass bins for the observed SMFs. Uncertainty in stellar mass measurements is accounted for according to equation (7). When comparing the predicted and observed SMFs, we jointly constrain the $\Phi_{\max }$ and $\Phi_{10}$ (referred to as $\Phi_{\text {obs }}$ ) by taking the measured covariance matrix $\left(\mathbf{C}_{\mathrm{obs}}\right)$ into account. The log-likelihood for SMF is:

$\ln \mathcal{L}_{\mathrm{SMF}}=-\frac{1}{2}\left[\Phi_{\text {mod }}-\Phi_{\text {obs }}\right]^{T} \mathbf{C}_{\text {obs }}^{-1}\left[\Phi_{\text {mod }}-\Phi_{\text {obs }}\right]+K$,

where $\Phi_{\text {mod }}$ is the predicted SMFs for $\mathcal{M}_{\star}^{10}$ and $\mathcal{M}_{\star}^{\text {Max }}$ aligned in the same order with the observed SMFs. $K$ is a constant described by $-\frac{1}{2}\left[\ln (2 \pi) N+\ln \left(\operatorname{det}\left(\mathbf{C}_{\text {obs }}\right)\right)\right]$ and $N=17$, which is the total number of mass bins.

The lensing observable, $\Delta \Sigma$, is computed directly from the simulation using 50 million randomly selected dark matter particles and the mock_observables.delta_sigma function in the halotools (Hearin et al. 2017). We predict the weightedmean $\Delta \Sigma$ profiles in the same 12 aperture mass bins used for observation for comparison after considering the uncertainties of the predicted $\mathcal{M}_{\star}^{10}$ and $\mathcal{M}_{\star}^{\text {Max }}$ into the weight. Our method accounts for the effects of scatter, the finite width of our bins, satellite galaxies, and the two-halo term. We ignore the contribution of $M_{\star}$ to $\Delta \Sigma$ because it is negligible on the scales that we consider $(r>200 \mathrm{kpc})$.

The log-likelihood for comparing $\Delta \Sigma$ profiles is described as:

$\ln \mathcal{L}_{\Delta \Sigma_{j}}=-\frac{1}{2} \sum_{i}^{n} \frac{\left(\Delta \Sigma_{\mathrm{mod}, \mathrm{i}}-\Delta \Sigma_{\mathrm{obs}, \mathrm{i}}\right)^{2}}{\sigma_{i}^{2}}+\sum_{i}^{n} \ln \left(2 \pi \sigma_{i}^{2}\right)$,

where the sum over $i$ is for $n=11$ radius bins of each $\Delta \Sigma$ profile and $\sigma_{i}$ is the associated observational uncertainty derived using a jackknife resampling method.

\section{RESULTS}

\subsection{Fitting our model to the data}

Finally, we combine the likelihood for SMF and $\Delta \Sigma$ profiles for the model:

$\ln \mathcal{L}_{\text {tot }}=\ln \mathcal{L}_{\mathrm{SMF}}+\sum_{j}^{m} \ln \mathcal{L}_{\Delta \Sigma_{j}}$

The sum over $j$ is for the $m=12$ aperture mass bins. To sample the posterior distributions of model parameters, we choose to use the affine invariant MCMC ensemble sampler EMCEE (ForemanMackey et al. 2013). We use an ensemble of 256 walkers. Following the strategy of the SED fitting code prospector (Leja et al. 2017; Johnson et al. in preparation), we separate the burn-in stage into three separated rounds, each with 150 steps. We reinitialize the walkers at the end of each round using the current best position of the ensemble and the covariance matrix measured using positions of 50 percent walkers. This method can effectively remove stalled walkers and helps the chains to converge. It also help us start the sampling chains from regions close to the highest probability position. We sample 9000 steps in this final run and use the Kullback-Leibler divergence $(\mathrm{K}$ $\mathrm{L}$ divergence) to empirically check the convergence of sampling chains every 100 steps. Following a similar procedure used in SED fitting tool prospector, we set the $\mathrm{K}-\mathrm{L}$ divergence threshold at 0.018 . We exclude the first 1000 steps and use the rest samples to form the final posterior distributions. According to the EMCEE document on the convergence and autocorrelation time $(\tau)$, one needs to sample at least $50 \times \tau$ steps to secure a fully converged chain. We should note that the current chains of our model have not met this strict standard yet. ${ }^{10}$ However, we exam the results from sampling runs with 3000 and 5000 steps, and find they are fully consistent with the one reported here. We also experiment with different 'moves' for the walker other than the default 'stretch' move provided by the updated version of EMCEE and also find consistent results. Therefore, we have reasons to believe that the model result presented here is stable and robust. The convergence analysis of our model can be found here: https://gith ub.com/dr-guangtou/asap/blob/master/note/convergence.ipynb and the trace plot of the final model is available here: https://gith ub.com/dr-guangtou/asap/blob/master/note/fig3_default.ipynb. We choose weakly informative priors for the seven parameters in our model. For the slopes of all the log-linear scaling relations in our model $\left(a, c\right.$, and $\left.A_{\text {exs }}\right)$, we adopt the Student- $t$ distribution with one degree of freedom as the priors to ensure the distribution of angle between the linear relation and the $x$-axis is sampled uniformly (e.g. Dose 2003; Sharma 2017). For other parameters ( $b, d, f_{\text {ins }}$, and $B_{\text {exs }}$ ), we choose simple top-hat distributions with reasonable boundaries. For instance, the upper limit for in situ stars within inner $10 \mathrm{kpc}$ is naturally 1.0 . We summarize the prior distributions of all seven parameters in the upper-right table of Fig. 3. Different choices of prior distributions (e.g. top-hat distributions for all parameters) does not alter key conclusions of this work.

\footnotetext{
${ }^{10}$ As we will show later, the current UniverseMachine output and model assumption present us from meaningfully constraining the parameters regarding the scatter of the TSHMR $(c$ and $d$ ). This may affect the convergence of the final sampling chain.
} 


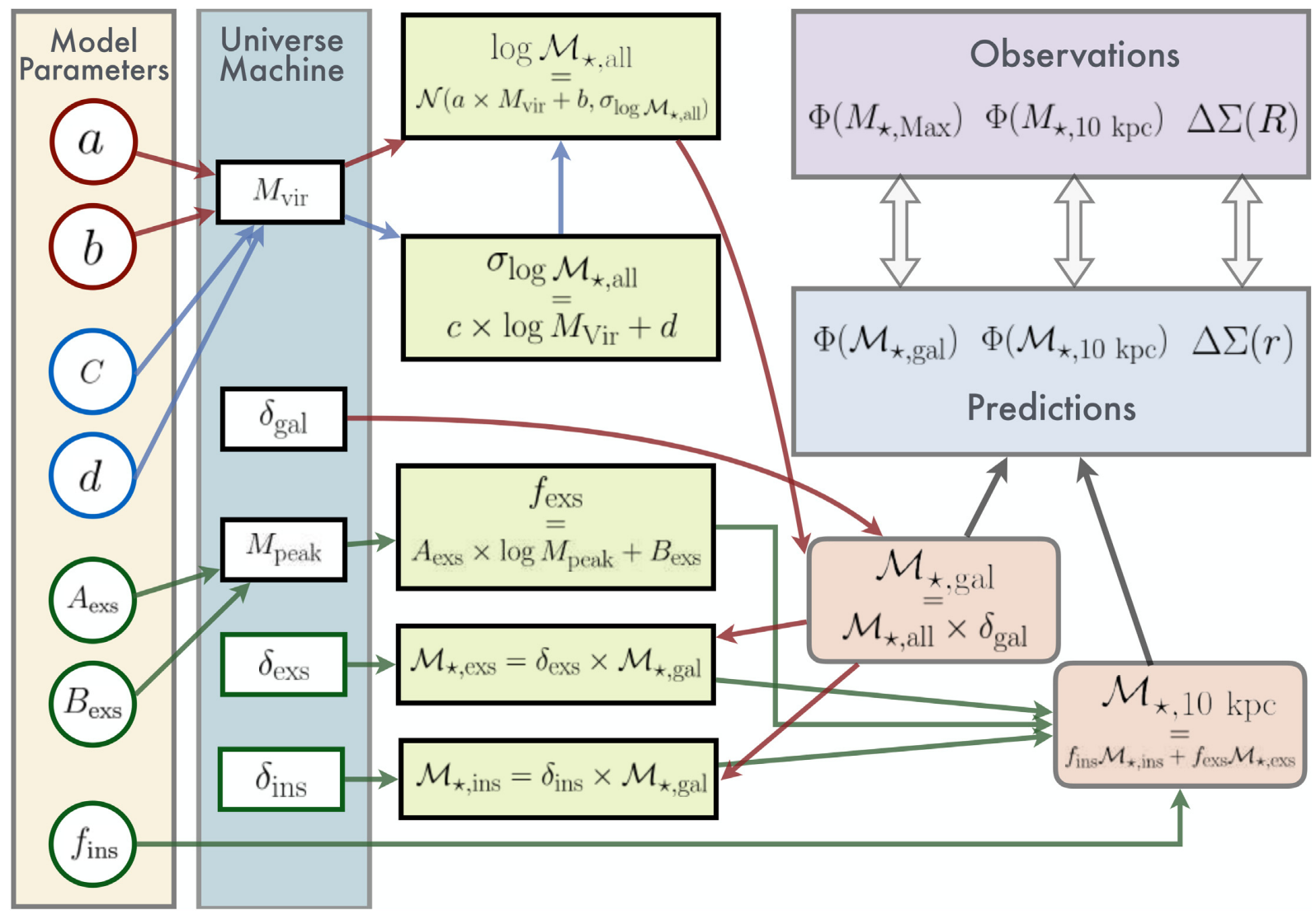

Figure 2. Flowchart for the basic design of the ASAP model. The UniverseMachine predictions adopted in this model are highlighted on the right. These correspond to: $M_{\text {peak }}$ - the peak halo mass; $\delta_{\text {gal }}$ - the ratio between the stellar mass of a galaxy $\left(M_{\star}^{\text {gal }}\right)$, and the total stellar mass within the halo $\left(M_{\star}^{\text {all }}\right)$; also the fraction of in situ $\left(\delta_{\text {ins }}\right)$ and ex situ $\left(\delta_{\text {exs }}\right)$ components in the $M_{\star}$ of each galaxy. The seven free model parameters are labelled on the bottom: $a$ and $b$ describe a $\log -\log$ linear relation between $M_{\text {peak }}$ and $M_{\star}^{\text {all }} ; c$ and $d$ describe a linear relation between the scatter of $M_{\star}^{\text {all }}$ and $M_{\text {peak. These four parameters, }}$ along with the $\delta_{\text {gal }}$ fraction predicted by UniverseMachine, provide predictions of the stellar mass of each galaxy $\left(\mathcal{M}_{\star}^{\text {gal }}\right)$ that will be compared with the observed $M_{\star}^{\text {max }}$. The stellar mass of in situ and ex situ components $\left(\mathcal{M}_{\star}^{\text {exs }}\right.$ and $\left.\mathcal{M}_{\star}^{\text {exs }}\right)$ are estimated using $\mathcal{M}_{\star}^{\text {gal }}, \delta_{\text {ins }}$, and $\delta_{\text {exs }}$. The predicted stellar mass in $10 \mathrm{kpc}\left(\mathcal{M}_{\star}^{10}\right)$ requires another three free parameters; $f_{\text {ins }}$ describes the fraction of in situ stars located within the inner $10 \mathrm{kpc}$, and the fraction of ex situ stars in $10 \mathrm{kpc}$ follows a linear relation with $\log _{10} M_{\text {peak }}$ that is characterized by $A_{\text {ins }}$ and $B_{\text {exs }}$. A keynote version of this flowchart is available here: https://github.com/dr-guangtou/asap/blob/master/doc/flowchart.key.

\subsection{Performance of the best-fitting model}

Here, we summarize the key results from our best-fitting model. Fig. 3 presents the best-fitting parameters along with their 68 percent confidence intervals. We show the two-dimensional marginalized probability densities of these parameters and the histograms of their marginalized posterior distributions using corner plots. ${ }^{11}$ The parameters are well-constrained. The correlations between $a$ and $b$, also between $A_{\text {exs }}$ and $B_{\text {exs }}$, are expected. As shown in Fig. 4, the best-fitting model is capable of reproducing the observations, including the SMFs for both $\mathcal{M}_{\star}^{\text {Max }}$ and $\mathcal{M}_{\star}^{10}$, and the $\Delta \Sigma$ profiles in different aperture mass bins. The predicted SMFs of $\mathcal{M}_{\star}^{10}$ and $\mathcal{M}_{\star}^{\mathrm{Max}}$ are consistent with the observed galaxies at $\log _{10}\left(M_{\star, \max } / \mathrm{M}_{\odot}\right)>11.6$ within uncertainties. And the predicted SMF of $\mathcal{M}_{\star}^{\text {Max }}$ is also consistent with the SMF from the PRIMUS survey at similar redshift range (Moustakas et al. 2013) down to $\log _{10}\left(M_{\star, \max } / \mathrm{M}_{\odot}\right) \sim 11.2$ where no observations are included. As for the $\Delta \Sigma$ profiles, the overall goodness-of-fit is excellent, although small mismatches can be found at $>1 \mathrm{Mpc}$ in a few aperture mass bins (e.g. bin 1, 2, and 12).

\footnotetext{
${ }^{11}$ Made by corner . py: https://corner.readthedocs.io/en/latest/.
}

\subsection{Best-fitting TSMR and SHMR}

\subsubsection{TSHMR}

From the best-fitting model, we have the TSHMR:

$$
\log \mathcal{M}_{\star}^{\text {all }}=0.589_{-0.002}^{+0.002} \times\left(\log M_{\text {vir }}-13.5\right)+11.844_{-0.001}^{+0.002} \text {. }
$$

We show the distribution of central galaxies on the $M_{\text {vir }}-\mathcal{M}_{\star}^{\text {all }}$ plane and the median TSHMR in panel (a) of Fig. 5. Fig. 5 also compares the best-fitting TSHMR with other observational constraints of groups and clusters at similar redshifts. Leauthaud et al. (2012b) constrain the TSHMR of groups in the COSMOS field at $0.22<z$ $<0.48$. Budzynski et al. (2014) derive the TSHMR for a large sample of low-redshift SDSS groups and clusters using optical richness. Patel et al. (2015) estimate the TSHMR for X-ray groups $\left(M_{200 c}<10^{13.5} \mathrm{M}_{\odot}\right)$ in the Chandra Deep Field South (CDF-S) field. The slope of our TSHMR $(0.602 \pm 0.005)$ is shallower than some previous estimates (e.g. $0.89 \pm 0.14$ in Budzynski et al. 2014; $0.84 \pm 0.10$ in Patel et al. 2015; also see Giodini et al. 2009; Lin et al. 2012; van der Burg et al. 2014), but once we convert different TSHMRs into $h=0.7$ with the Chabrier IMF, the overall agreement is good. And Kravtsov et al. (2018) measure the TSHMR for 21 massive $z \sim 0$ clusters using X-ray observations and 


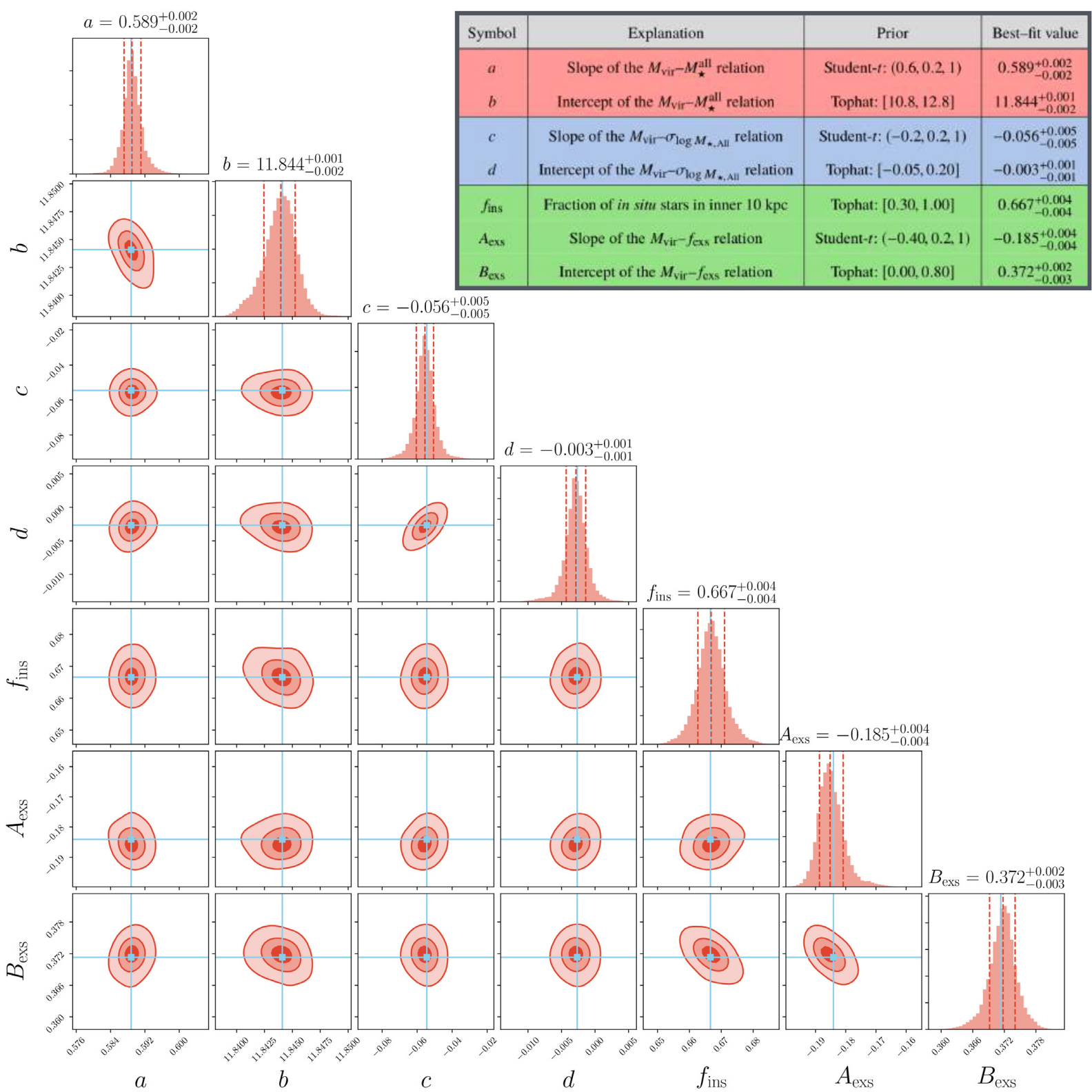

Figure 3. Corner plot for the posterior probability distributions of parameters in the model. The contour levels describe 16 per cent, 50 per cent, and 84 per cent, enclosed probability regions. On each histogram, the three dash lines highlight the mean value and the $1 \sigma$ range of the distribution. The explanations, ranges of uniform priors, and the best-fitting values along with their uncertainties are highlighted in the upper-right table. The colours separate parameters into three groups as indicated in the flowchart. The Jupyter notebook for this figure is available here: https://github.com/dr-guangtou/asap/blob/master/note/fig3_default.ipynb.

improved photometric models of massive brightest cluster galaxies (BCGs). The slope of the TSHMR using $M_{500 c}(0.59 \pm 0.08)$ is in good agreement with the result presented here. Our TSHMR is constrained by the deepest imaging data set for a large sample of massive galaxies and high signal-to-noise g-g lensing measurements. The best-fitting relation is consistent with other observational constraint down to $\log _{10} M_{\mathrm{vir}} \geq 12.5$, which extends below the halo mass range probed by the observed massive galaxies. Meanwhile, we notice that the best-fitting $d$ suggests that there is no scatter of TSHMR at $\log _{10} M_{\text {vir }} \geq 13.5$. This is clearly unphysical. It indicates that our current model cannot constrain the scatter of TSHMR. In this work, we assume the TSHMR is a more fundamental relation and should have smaller scatter than the SHMR for central galaxies. Several previous works support this assumption and report a very tight TSHMR (scatter <0.1 dex; e.g. van der Burg et al. 2014; Patel et al. 2015; Kravtsov et al. 2018). Meanwhile, we do not directly constrain the scatter of TSHMR but rely on the dark matter simulation and the UniverseMachine model to recover it based on the scatter of the observationally constrained SHMR. In this framework, the scatter of the observed SHMR should contain two parts: (1) the scatter of TSHMR (scatter of $M_{\star}^{\text {all }}$ at fixed $M_{\text {vir }}$ ) and (2) the scatter of $M_{\star}^{\text {cen }}$ at fixed $M_{\star}^{\text {all }}$. However, the current version of UniverseMachine displayed a very large scatter for the second part at $z \sim 0.4$ (please see Appendix A for details). Such scatter alone is already comparable or even larger than the observed scatter of SHMR, leaving no space to constrain the scatter of TSHMR. There are two possible explanations: (1) we underestimate the scatter of SMHR or (2) the UniverseMachine model overpredicts the 


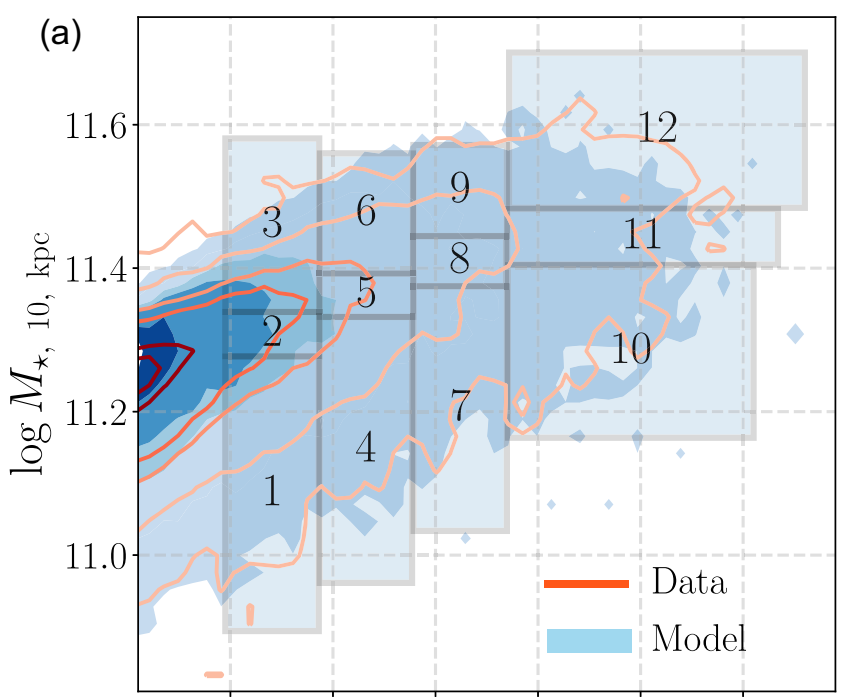

$\begin{array}{llllll}11.6 & 11.7 & 11.8 & 11.9 & 12.0 & 12.1\end{array}$ $\log M_{\star, \operatorname{Max}}$

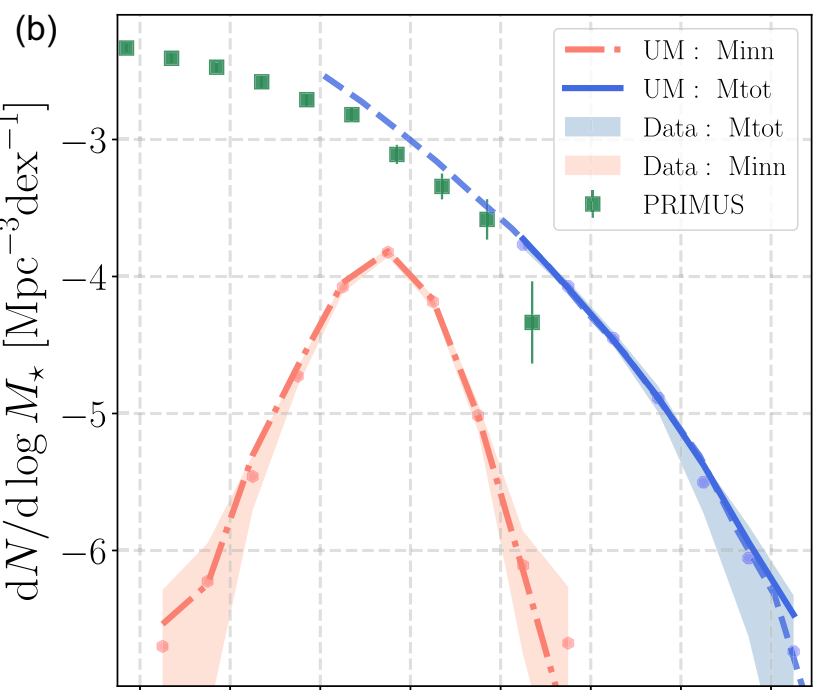

$\begin{array}{llllllll}10.8 & 11.0 & 11.2 & 11.4 & 11.6 & 11.8 & 12.0 & 12.2\end{array}$ $\log \left(M_{\star} / M_{\odot}\right)$

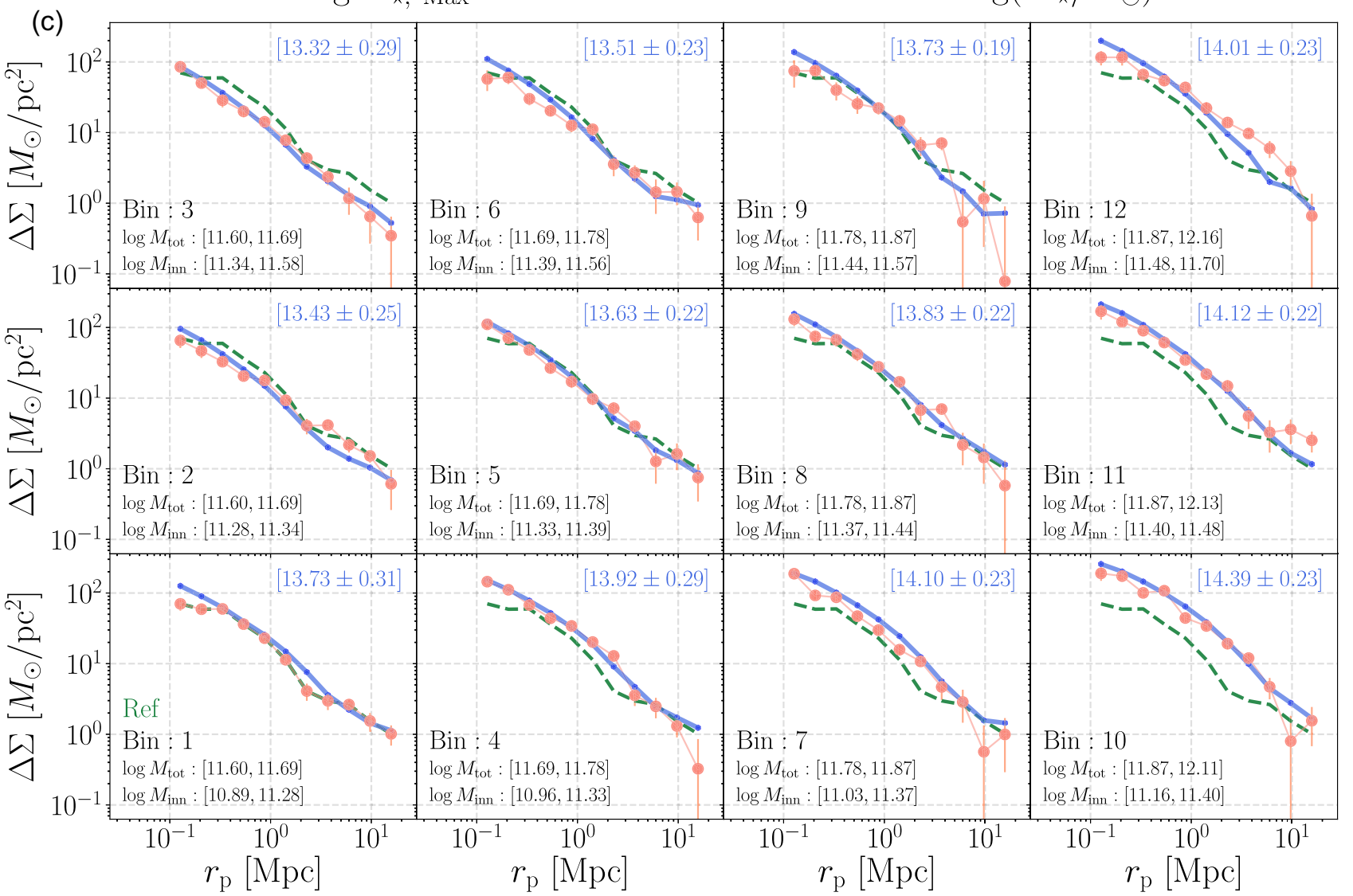

Figure 4. Performance of the best-fitting model. (a) Comparison between the distributions of observed HSC galaxies (open red contour) and modelled galaxies (solid blue contour) over the $M_{\star}^{\max }-M_{\star}^{10}$ plane. Both observed and modelled galaxies with $\log _{10}\left(M_{\star}, \max / \mathrm{M}_{\odot}\right) \geq 11.6$ are grouped into the same 12 bins using $M_{\star}^{\max }$ and $M_{\star}^{10}$ values to compare the g-g lensing signals within each bin. (b) Comparisons of observed (dots and shaded regions) and modelled SMFs (solid lines) for $M_{\star}^{\max }$ (blue) and $M_{\star}^{10}$ (red). We also overplot the SMF from the PRIMUS survey at similar redshift to show the shape of SMF at lower $M_{\star}$. (c) Comparisons of g-g lensing signals in each $M_{\star}^{\max }-M_{\star}^{10}$ bin. The bin number and the mass range of $M_{\star}^{\max }$ and $M_{\star}^{10}$ of each bin is given in the lower-left corner of each subplot. The observed g-g lensing signals are shown as red points, while the blue lines show the modelled lensing signal. The weak lensing signal from Bin $=1$ (bottom-left plot) is shown in each subplot as a green dashed line to highlight the evolution of $\Delta \Sigma$ amplitudes across various bins. The median $M_{\mathrm{vir}}$ in each bin is shown in the upper-right corner of each subplot. The Jupyter notebook for this figure is available here: https://github.com/dr-guangtou/asap/blob/master/note/fig4.ipynb. 
(a)

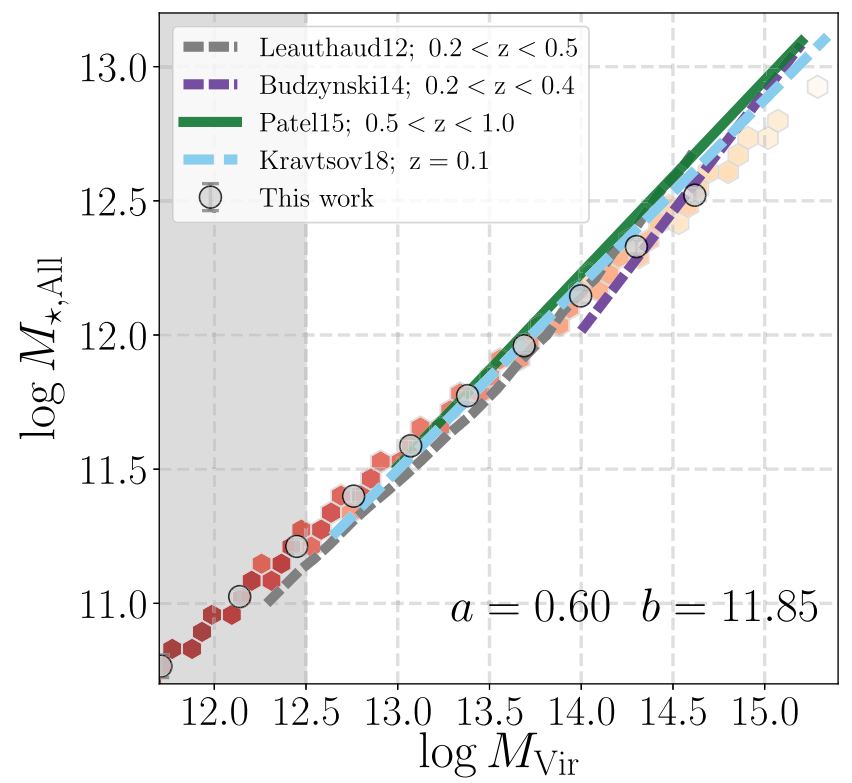

(b)

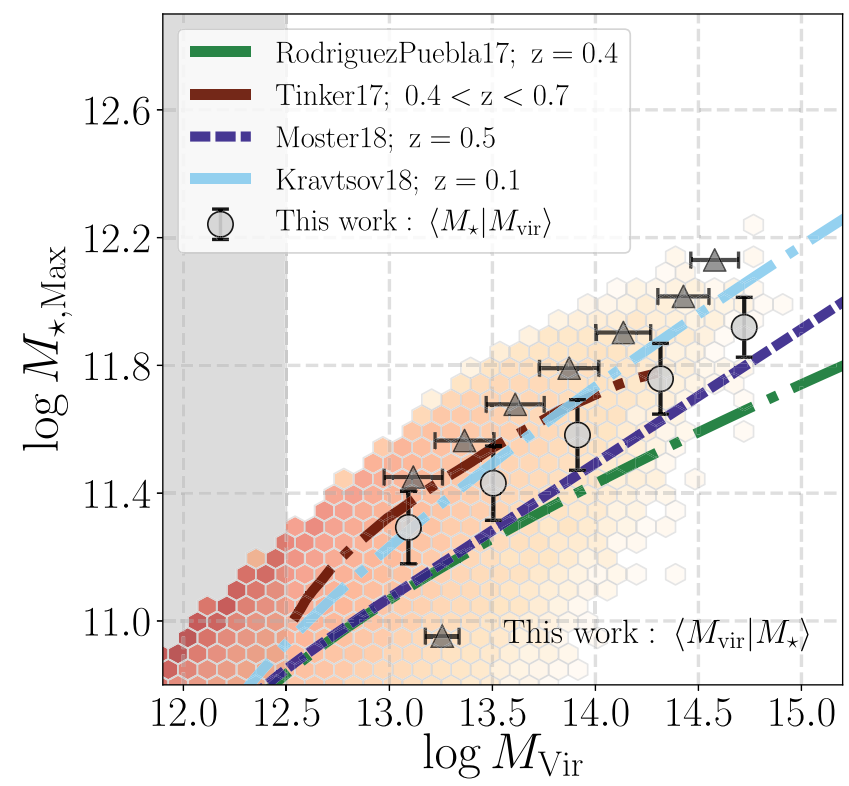

Figure 5. (a) Comparison between the TSHMR from our model and other work. The background density plots show the distributions of modelled galaxies where the colour indicates the number density of galaxies. TSHMR from literature includes Leauthaud et al. 2012b (dashed grey line), Budzynski et al. 2014 (dashed purple line), Patel et al. 2015 (solid green line), and Kravtsov et al. 2018 (dashed teal line). Our median TSHMR is highlighted by grey circles. (b) Comparison between the SHMR of central galaxies from our best-fitting model and recently published SHMRs at similar redshifts, including Rodríguez-Puebla et al. (2017) (dot-dashed green line), Tinker et al. (2017) (dot-dashed brown line), Moster et al. (2018) (dashed purple line), and Kravtsov et al. (2018) (solid pink line). For the median SHMR: the grey circles show the median $M_{\star}^{\max }$ at different $M_{\star}^{\max }$ bins while the grey triangles show the median $M_{\mathrm{vir}}$ at fixed $M_{\star}^{\max }$ Error bars indicate the scatter of $M_{\star}^{\max }$ or $M_{\mathrm{vir}}$ within the bin. All comparisons are under the same fiducial assumptions of $h$-factor $h=0.7$, Chabrier IMF, and FSPS stellar population models. The Jupyter notebook for this figure is available here: https://github.com/dr-guangtou/asap/blob/master/note/fig5.ipynb.

scatter of $M_{\star}^{\text {cen }}$ at fixed $M_{\star}^{\text {all }}$. The first explanation is possible since we only have a handful of galaxy-galaxy lensing profiles at the high- $M_{\star}^{\text {cen }}$ end to constrain the SHMR. But this is unlikely. As we will show in the next section, the scatter of the 'best-fitting' SHMR is comparable to many recent constraints based on very different data or modelling approaches. We see no evidence that we underestimate the scatter of SHMR at the high- $M_{\text {vir }}$ end. Therefore, we think the fact we failed to constrain the scatter of TSHMR hints an issue with the current UniverseMachine model. Fig. A1 shows that the scatter of $\delta_{\mathrm{Cen}}\left(\right.$ or $\left.M_{\star}^{\text {cen }}\right)$ significantly increase with decreasing $M_{\star}^{\text {all }}$ (or $M_{\mathrm{vir}}$ ). Such scatter is clearly large enough to fully account for the scatter of SHMR. At the same time, it suggests that the UniverseMachine output now contains a population of massive central galaxies $\left(\log _{10}\left(M_{\star} / \mathrm{M}_{\odot}\right)>11.5\right)$ living in relative less massive dark matter haloes (e.g. $\log _{10} M_{\text {vir }} \sim 13.0$ ) that completely dominate the $M_{\star}$ budget of the halo $\left(\delta_{\mathrm{Cen}}>0.8\right)$. We think this is not likely to be real and reflect a limitation of the current UniverseMachine model. It may relate to the artificial disruption of small haloes in $\mathrm{N}$-body simulations due to limited resolution (e.g. van den Bosch et al. 2018; van den Bosch \& Ogiya 2018) and how does UniverseMachine handles the disruption of satellite galaxies in massive haloes, but such discussion is beyond the scope of this work. We will investigate this further and iterate with future versions of the UniverseMachine model.

\subsubsection{SHMR}

Fig. 5(b) displays the number density distribution of model galaxies (indicated by colour) over the $M_{\mathrm{vir}}-M_{\star}^{\mathrm{max}}$ plane. As discussed in Tinker et al. (2017) and Rodríguez-Puebla et al. (2017), the relations of $\left\langle M_{\star}\right\rangle_{M_{\mathrm{vir}}}$ (SHMR described by the mean $M_{\star}^{\max }$ at fixed $M_{\mathrm{vir}}$; grey circles) and $\left\langle M_{\mathrm{vir}}\right\rangle_{M_{\star}}$ (SHMR using the mean $M_{\mathrm{vir}}$ in bins of $M_{\star}^{\max }$; grey triangles) have different slopes and scatters. Therefore we show these two relations separately. A log-linear fit for $\left\langle M_{\star}\right\rangle_{M_{\mathrm{vir}}}$ at $\log M_{\text {vir }} \geq 13.0$ yields:

$\log M_{\star}^{\max }=0.36 \pm 0.01 \times\left(\log M_{\text {vir }}-13.27\right)+11.38 \pm 0.02$

with a scatter of $\sigma_{\log M_{\text {vir }}}=0.23 \pm 0.01$. The best-fitting log-linear relation for $\left\langle M_{\mathrm{vir}}\right\rangle_{M_{\star}}$ at $\log M_{\star}^{\max } \geq 11.5$ is:

$\log M_{\text {vir }}=2.49 \pm 0.02 \times\left(\log M_{\star}^{\max }-11.6\right)+13.39 \pm 0.02$

with a scatter of $\sigma_{\log M_{\star}^{\max }}=0.22 \pm 0.01$. We compare our results with recent constraints of SHMR in the form of $\left\langle M_{\star}\right\rangle_{M_{\mathrm{vir}}} .{ }^{12}$ Tinker et al. (2017) estimate the SHMR for massive $\left(\log M_{\star} \geq 11.4\right)$ CMASS galaxies (e.g, Dawson et al. 2013) at $0.4<z<0.7$ using clustering measurements. The SHMR from Kravtsov et al. (2018) shown here is from an abundance matching method based on the SMFs from Bernardi et al. (2013). It is worth noting that the $M_{\star}$ used in above works are based on different images and photometric methods. For instance, $M_{\star}$ in Kravtsov et al. (2018) are based on extrapolating profiles of massive galaxies to infinity, therefore they are expected to be larger than the equivalent $M_{\star}^{\max }$ measurements. The impact of different choices of photometry on the slope and scatter of SHMR will be investigated more carefully in the near

\footnotetext{
${ }^{12}$ All SHMR have also been converted to $h=0.7$, Virial halo mass and the Chabrier IMF.
} 
future (Huang et al. in preparation). The SHMRs of RodríguezPuebla et al. (2017) and Moster et al. (2018) are from two new semi-empirical models that are similar to the UniverseMachine in methodology. Recent empirical models (e.g. Rodríguez-Puebla et al. 2017; Moster et al. 2018; Behroozi et al. 2019 have adopted the improved $z \sim 0$ SMF from Bernardi et al. 2013) which uses a better background subtraction. This approach could lead to better agreement with our result using deep HSC images than earlier models that are constrained by local SMFs that underestimate the masses of massive galaxies (e.g. Behroozi et al. 2013b). Scatter in the SHMR includes an intrinsic component and uncertainties of stellar mass measurements. Our results agree well with recent constraints when described by $\sigma_{\log M_{\star}}$ at fixed $M_{\text {vir }}$. Tinker et al. (2017) find $\sigma_{\log M_{\star}}=0.18_{-0.02}^{+0.01}$ at $\log M_{\star} \geq 11.4$. The Emerge model by Moster et al. (2018) shows a scatter of $\sigma_{\log M_{\star}}=0.16$ at high masses. In Kravtsov et al. (2018), the authors explicitly measured a scatter of $0.17 \pm 0.03$ dex for $M_{\star, B C G}$ at fixed $M_{500 c}$ using 21 clusters. Along with other recent work (e.g. Reddick et al. 2013; Zu \& Mandelbaum 2016), these estimates leave little room for intrinsic scatter in the high-mass SHMR $\left(\sigma_{\log M_{\star}}^{\operatorname{intr}}<0.16\right)$. In $\mathrm{Gu}$, Conroy \& Behroozi (2016), the authors used a simple toy model based on $N$-body simulation to show that a similar amount of scatter $(\sim 0.2$ dex $)$ can naturally emerge from the complex merging history of massive haloes thanks to the central limit theorem. Under our model assumptions, the scatter of SHMR consists of both the scatter of the TSHMR and the scatter of $\delta_{\text {Cen }}$ at fixed $M_{\star}^{\text {all }}$. Both of these components should reflect certain aspects of the halo and stellar mass assembly history. Unfortunately, the current UniverseMachine model and HSC observations do not enable us to look into the scatter of TSHMR and the scatter of $\delta_{\mathrm{Cen}}$ at fixed $M_{\star}^{\text {all }}\left(\right.$ or $M_{\text {vir }}$ ) completely dominates the scatter of SHMR. We will further look into the issues about scatters of TSHMR and SHMR using alternative models and new observations.

\subsection{Variations of $M_{\text {vir }}$ across the $M_{\star}^{\max }-M_{\star}^{10}$ plane}

The main goal of our model is to evaluate the $M_{\star}^{\max }-M_{\star}^{10}-M_{\mathrm{vir}}$ relation. Fig. 6 displays variations in $M_{\text {vir }}$ across the aperture mass plane (A 3D interactive visualization is here: https://plot.ly $/ \sim$ shuan $\mathrm{g} 89 / 1 /$ ). This trend is strongly constrained by the $\Delta \Sigma$ profiles in different aperture mass bins. The variation is also consistent with the intuition we initially gained from Fig. 4. The amplitude of $\Delta \Sigma$ increases with $M_{\star}^{\max }$ but also decreases with $M_{\star}^{10}$ at fixed $M_{\star}^{\max }$. This indicates higher $M_{\text {vir }}$ for massive galaxies with more extended stellar envelopes. The median $M_{\text {vir }}$ in each $M_{\star}^{\max }-M_{\star}^{10}$ bin is shown on the upper-right corner of each subplot of panel (c) in Fig. 4. Typically, the range of $M_{\text {vir }}$ across the three bins with similar $M_{\star}^{\max }$ is about $0.15-0.20$ dex. But, as shown in panel (a) of Fig. 4, this is caused by the choices of $M_{\star}^{10}$ bins at fixed $M_{\star}^{\text {max }}$ : although they cover very different ranges of $M_{\star}^{10}$, the mean $M_{\star}^{10}$ values for the three bins are not very different due to the distribution of massive galaxies. Right now the choice of mass bins is limited by the required number of galaxies to ensure sufficient $\mathrm{S} / \mathrm{N}$ of the $\Delta \Sigma$ profile and uncertainties of stellar mass measurements $(\sim 0.1 \mathrm{dex})$. This is not ideal for direct measurement of 'local' $M_{\text {vir }}$ across the aperture mass plane and is precisely why we choose to use the forward modelling approach by simultaneously considering twelve $\Delta \Sigma$ profiles and two SMFs so that we can still use the best-fitting model to explore the $M_{\text {vir }}$ trend in more detail. The iso- $M_{\text {vir }}$ curves on Fig. 6 run almost parallel to the $M_{\star}^{\max }-M_{\star}^{10}$ relation, resulting in a considerable range of $M_{\mathrm{vir}}$ (>0.7 dex) in the vertical direction at fixed $M_{\star}^{\max }$. This range is not surprising, however, given the range of $M_{\mathrm{vir}}$ seen on the SHMR at fixed $M_{\star}^{\max }$ (e.g. see Fig. 5; also see fig. 9 in Tinker et al. 2017). One of the main results of this work is that the structural details of the surface density profiles of massive galaxies contain significant additional information about their dark matter haloes. Combining the $M_{\star}^{\text {max }}$ with other structural information (e.g. $M_{\star}^{10}$ ), we can obtain a better proxy of $M_{\mathrm{vir}}$. In other terms, the scatter in $M_{\mathrm{vir}}$ in greatly reduced in the aperture mass plane compared to the SHMR. Fig. 7 displays the scatter of $M_{\text {vir }}\left(\sigma_{\log M_{\text {vir }}}\right)$ across the aperture mass plane. Among regions occupied by most massive galaxies (indicated by the contours), the typical scatter is only of order 0.15 dex.

Fig. 7 suggests that the combination of $M_{\star}^{\text {max }}-M_{\star}^{10}$ predicts $M_{\text {vir }}$ better than $M_{\star}^{\max }$ alone. For instance, a simple random forest regressor ${ }^{13}$ can provide an accurate description of the $M_{\star}^{\max }-M_{\star}^{10}$ $M_{\text {vir }}$ 3D space and can be used to predict $M_{\text {vir }}$ (see Appendix B). However, with random forest there is a risk of overfitting, and the results are not intuitive. We therefore also fit the $M_{\star}^{\max }-M_{\star}^{10}$ $M_{\text {vir }}$ plane using the robust linear regression algorithm LtsFit (Cappellari 2014). The best-fitting relation is:

$$
\begin{aligned}
\log M_{\text {vir }}= & 3.26 \pm 0.02 \times\left(\log M_{\star}^{\max }-11.72\right) \\
& -2.46 \pm 0.03 \times\left(\log M_{\star}^{10}-11.34\right)+13.69 \pm 0.01
\end{aligned}
$$

with a scatter of $\sigma \log M_{\mathrm{vir}}=0.16 \pm 0.01$. As shown in Appendix B, this simple relation is also capable of predicting $M_{\text {vir }}$ with reasonable precision and a smaller scatter than the SHMR. We further discuss predictive capabilities in Section 7.2.

\subsection{In situ and ex situ fractions}

Since the version of UniverseMachine used here predicts the $M_{\star}$ of the in situ and ex situ components, our model can be used to shed light on the statistical behaviours of these two components. The best-fittin model suggests that $67 \pm 1$ percent of in situ stars can be found within $10 \mathrm{kpc}$, while the fraction of ex situ stars within $10 \mathrm{kpc}$ slowly decreases with $M_{\text {vir }}$ due to the increasingly extended distribution of the ex situ component. At $M_{\mathrm{vir}}=10^{13} \mathrm{M}_{\odot}$, about half of the ex situ stars lie inside $10 \mathrm{kpc}$ according to the best-fitting model. This fraction decreases to $\sim 30$ per cent for a $M_{\text {vir }}=10^{14} \mathrm{M}_{\odot}$ halo. Focusing on the ex situ component, we show how the fraction of ex situ stars changes with $M_{\star}^{\max }$ and $M_{\text {vir }}$ in Fig. 8. In agreement with results from recent hydrosimulations (e.g. Rodriguez-Gomez et al. 2016; Qu et al. 2017; Pillepich et al. 2018b), the ex situ fraction increases with both stellar and halo mass, and it remains the dominant stellar component in massive galaxies. Remarkably, this is not just the case for the galaxy as a whole, but it is even true on $10 \mathrm{kpc}$ scale for these massive galaxies. For central galaxies with $\log _{10}\left(M_{\star, \max } / \mathrm{M}_{\odot}\right)>11.5$ and in haloes with $\log _{10} M_{\mathrm{vir}}>13.5$, the average ex situ fraction at $r<10 \mathrm{kpc}$ is $>50$ percent. In Fig. 8, we also compare the trends of the ex situ fraction with halo mass with results from the IllustrisTNG simulation (Pillepich et al. 2018b; TNG hereafter). We find reasonable qualitative agreement between our model and the TNG simulation; differences in detail are to be expected, given the different methods used for measuring $M_{\star}$ (Ardilla et al. in preparation), e.g. for the ex situ fraction within $10 \mathrm{kpc}$, Pillepich et al. (2018b) use a 3D sphere while we use a 2D elliptical aperture. The dominant role of ex situ stars at the centres of

\footnotetext{
${ }^{13}$ E.g. The RandomForestRegressor from scikit-learn package. Random forest is a flexible machine-learning algorithm that uses a combinations of multiple decision trees to make predictions based on the data.
} 


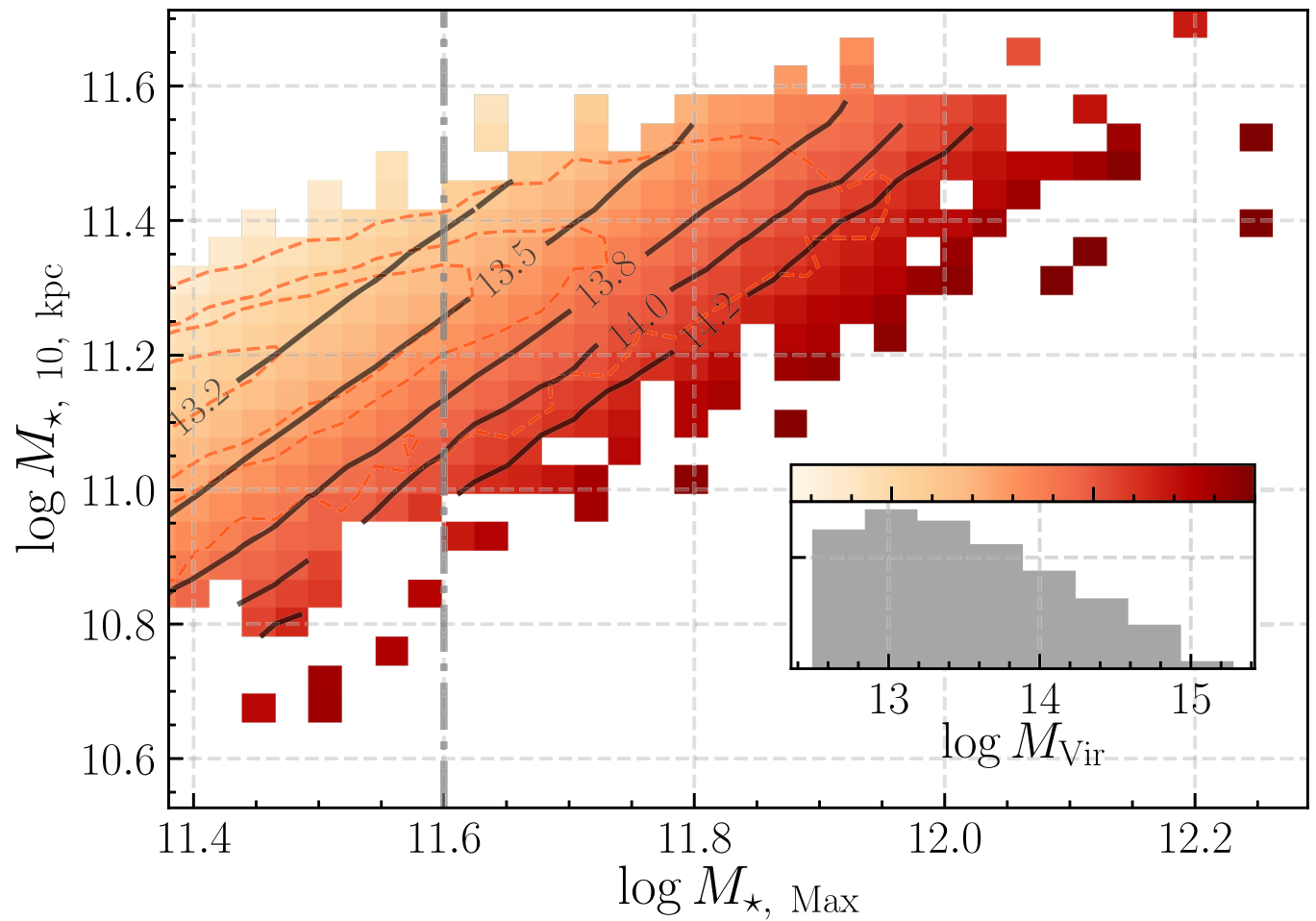

Figure 6. Variation of halo mass across the aperture mass plane based on the best-fitting ASAP model for massive central galaxies. Contours outlined using reddashed lines show the distribution of HSC galaxies. The underlying colour-gradient visualize the trend of $M_{\text {vir }}$ from the 'best-fitting' model. Solid-black lines with labels show the 'iso- $M_{\text {vir }}$ ' curves over the aperture mass plane. A dot-dashed line highlights the $M_{\star}^{\text {max }}$ limit used in our model. And we also show a histogram of predicted $M_{\text {vir }}$ for our massive galaxy sample. The Jupyter notebook for this figure is available here: https://github.com/dr-guangtou/asap/blob/master/note/f ig6.ipynb. And an interactive 3D plot of the $M_{\mathrm{vir}}-M_{\star}^{\mathrm{max}}-M_{\star}^{10}$ plane can be found here on-line: https://github.com/dr-guangtou/asap/blob/master/note/fig6.ipynb.
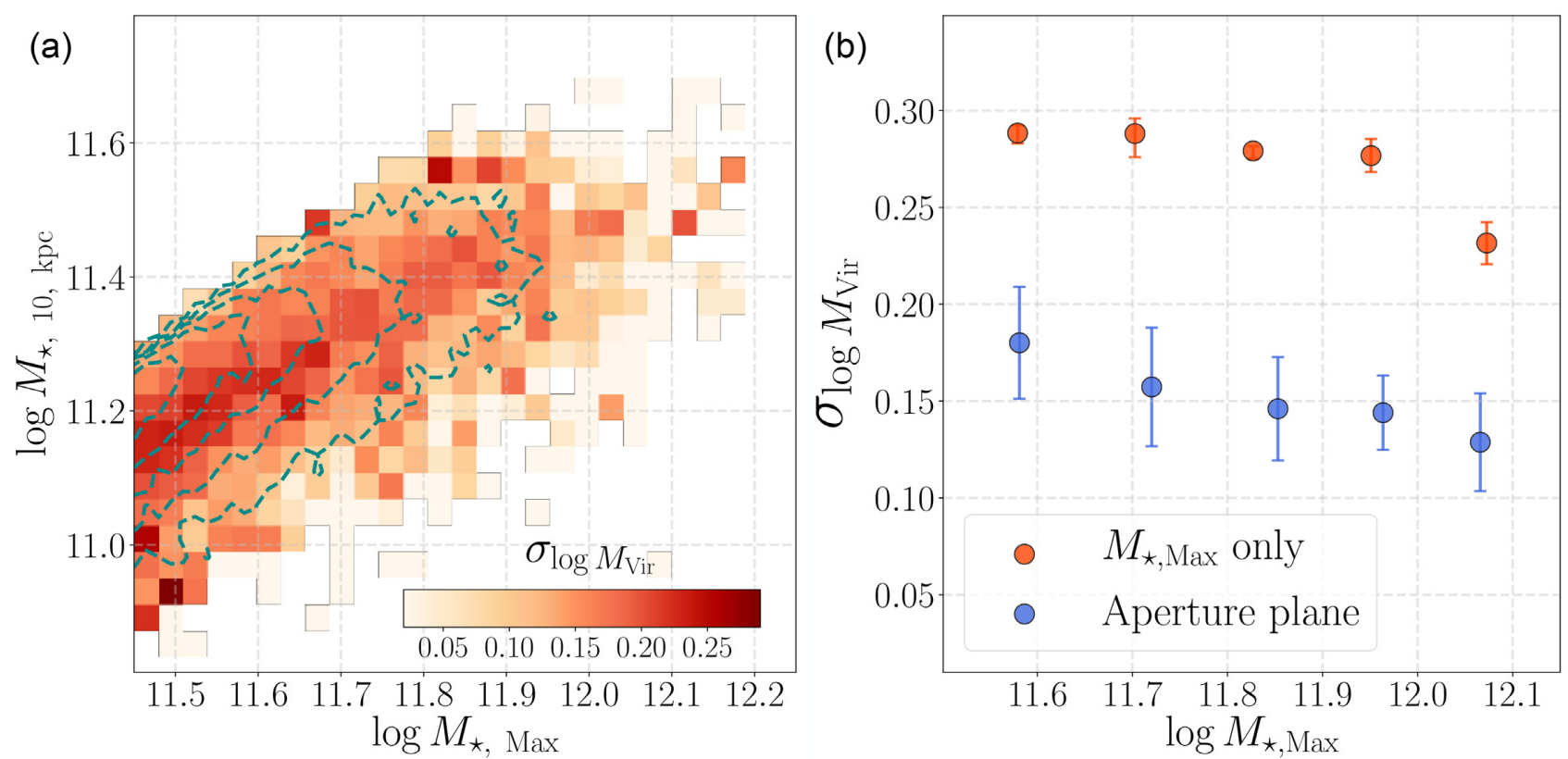

Figure 7. (a) Scatter of $\log _{10} M_{\text {vir }}$ across the aperture mass plane. The distribution of HSC galaxies is outlined using contours (grey dashed line). (b) Scatter of $\log _{10} M_{\mathrm{vir}}$ at different $M_{\star}^{\max }$. Red points correspond to the scatter in different $M_{\star}^{\max }$ bins. Blue points show the mean scatter when information from the aperture mass plane is considered. The errors bars are estimated by randomly drawing from the posterior distributions of model parameters. Scatter here includes both an intrinsic component as well as the uncertainty of stellar mass measurements. The Jupyter notebook for this figure is available here: https://github.com/dr-guangtou/asap/blob/master/note/fig7.ipynb. 

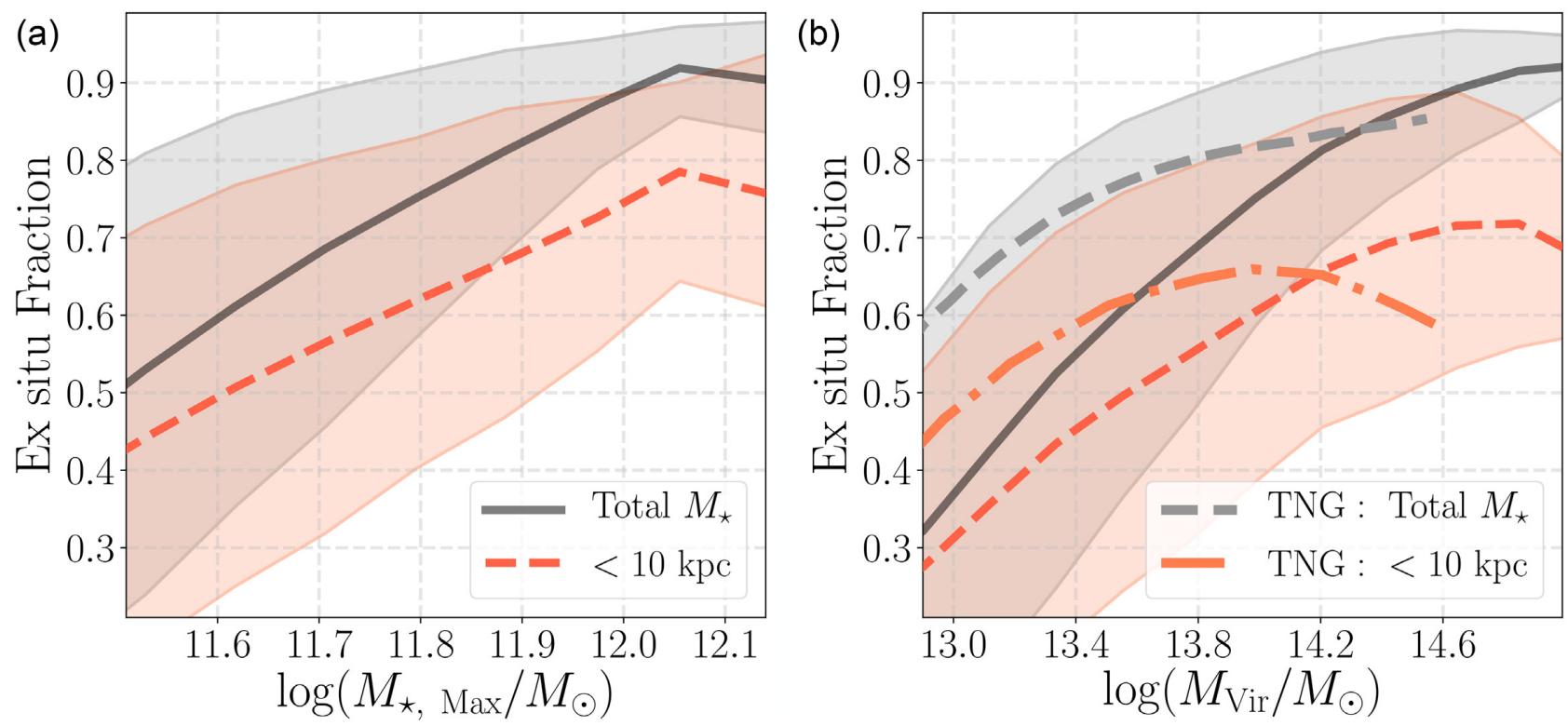

Figure 8. (a) The relation between the fraction of ex situ stars and stellar mass ( $\left.M_{\star}^{\max }\right)$ using the best-fitting ASAP model. (b) The same relation between halo mass $\left(M_{\mathrm{vir}}\right)$. Solid black lines and the corresponding shaded regions are for the ex situ fraction in the total stellar mass, while the dashed-red lines and the associated shaded regions indicate the ex situ fraction within $10 \mathrm{kpc}$. The shaded regions describe the $1 \sigma$ uncertainties. In massive galaxies, ex situ stars dominate the total stellar mass budget and the central stellar mass when $\log _{10}\left(M_{\star, \max } / \mathrm{M}_{\odot}\right)>11.5$ or when $\log _{10} M_{\text {vir }}>13.5$. In (b) we also compare our results with similar relations from the TNG300 simulation (see Pillepich et al. 2018b for details). The Jupyter notebook for this figure is available here: https://github.com/dr-guangtou/asap/blob/master/note/fig8.ipynb.

massive galaxies has been discussed by Cooper et al. (2013) using a particle-tagging method and by Rodriguez-Gomez et al. (2016) using the Illustris simulation. It is likely that these ex situ stars originate from major mergers that happened at high- $z$. This is directly related to the current definition of the in situ and ex situ components. We discuss this further in Section 7.3.

\subsection{Relation between $M_{\text {vir }}$ and galaxy size}

Fig. 9 shows variations in the mean $M_{\text {vir }}$ across the mass-size relation. Here we use $M_{\star}^{\max }$ and a non-circularized half-light radius measured using a 1D stellar mass curve of growth. For each massive galaxy, we assign a $M_{\text {vir }}$ using the best-fitting $M_{\star}^{\max }-M_{\star}^{10}-M_{\text {vir }}$ relation derived above. We do not attempt to remove satellite galaxies. In Paper I, we showed that massive central galaxies in haloes of different mass exhibit a distinct mass-size relation. Panel (b) of Fig. 9 presents more sophisticated constraints on this 'environmental' dependence of mass-size relation: $M_{\mathrm{vir}}$ varies systematically across this plane, and the iso- $M_{\text {vir }}$ curves here are almost perpendicular to the mass-size relation. At fixed $M_{\star}^{\max }$, galaxies with larger size tend to live in more massive haloes. This suggests that the sizes of massive galaxies also carry clues about their dark matter halo mass. For instance, as suggested by Kravtsov (2013), there is a log-linear relation between the virial radius of the halo and the half-light radius of the galaxy (For this relation for our sample, please see the notebook here: https://github.com/dr-guan gtou/asap/blob/master/note/assign_halo_mass.ipynb). However, as discussed in Paper I, the measurement of 'galaxy size' often depends on the assumed photometric model and data quality. Therefore, we prefer to build our empirical model based on a more straightforward aperture mass plane instead of the mass-size relation.

\section{DISCUSSION}

\subsection{Comparison with hydrodynamic simulations}

From HSC g-g lensing measurement and our best-fitting model, we find that the halo masses of massive galaxies vary systematically across the aperture mass plane. This reveals a clear connection between the distribution of stars within massive galaxies, and halo mass. We now investigate if such correlations are also predicted by hydrodynamic simulations of galaxy evolution. We compare the observed $M_{\star}^{\max }-M_{\star}^{10}-M_{\mathrm{vir}}$ relation with the relations of massive galaxies from the MassiveBlackII simulation (e.g. Khandai et al. 2015; Tenneti et al. 2015). Mass iveBlackII is a state-of-the-art, large-volume (100 $\mathrm{h}^{-1} \mathrm{Mpc}$ box size; $1792^{3}$ gas particles), highresolution cosmological simulation using $\mathrm{p}$-Gadget (Springel 2005). It includes a sophisticated treatment of complex baryonic physics (e.g. star formation in a multiphase interstellar medium, black hole accretion and feedback, and radiative cooling and heating processes). For additional information about the physical details and general performance of the MassiveBlackII simulation, please refer to Khandai et al. (2015). We select 291 massive galaxies with $\log \left(M_{\star} / \mathrm{M}_{\odot}\right) \geq 11.4$ from the MassiveBlackII simulation and generate randomly projected $2 \mathrm{D}$ stellar mass maps with a $2 \mathrm{kpc}$ pixel resolution and $350 \mathrm{kpc}$ image size. Then we treat them as real data and measure their aperture masses using the same method for HSC massive galaxies (Ardila et al. in preparation). We choose to use 10 and $100 \mathrm{kpc}$ elliptical apertures here. In Fig. 10, we show the trend of halo mass across this aperture mass plane recovered by the locally weighted regression (LOESS) method (Cleveland \& Devlin 1988; Cappellari et al. 2013). ${ }^{14}$ While the slope of the iso-

\footnotetext{
${ }^{14}$ We use the PYTHON implementation of 2D LOESS by Michelle Cappellari.
} 

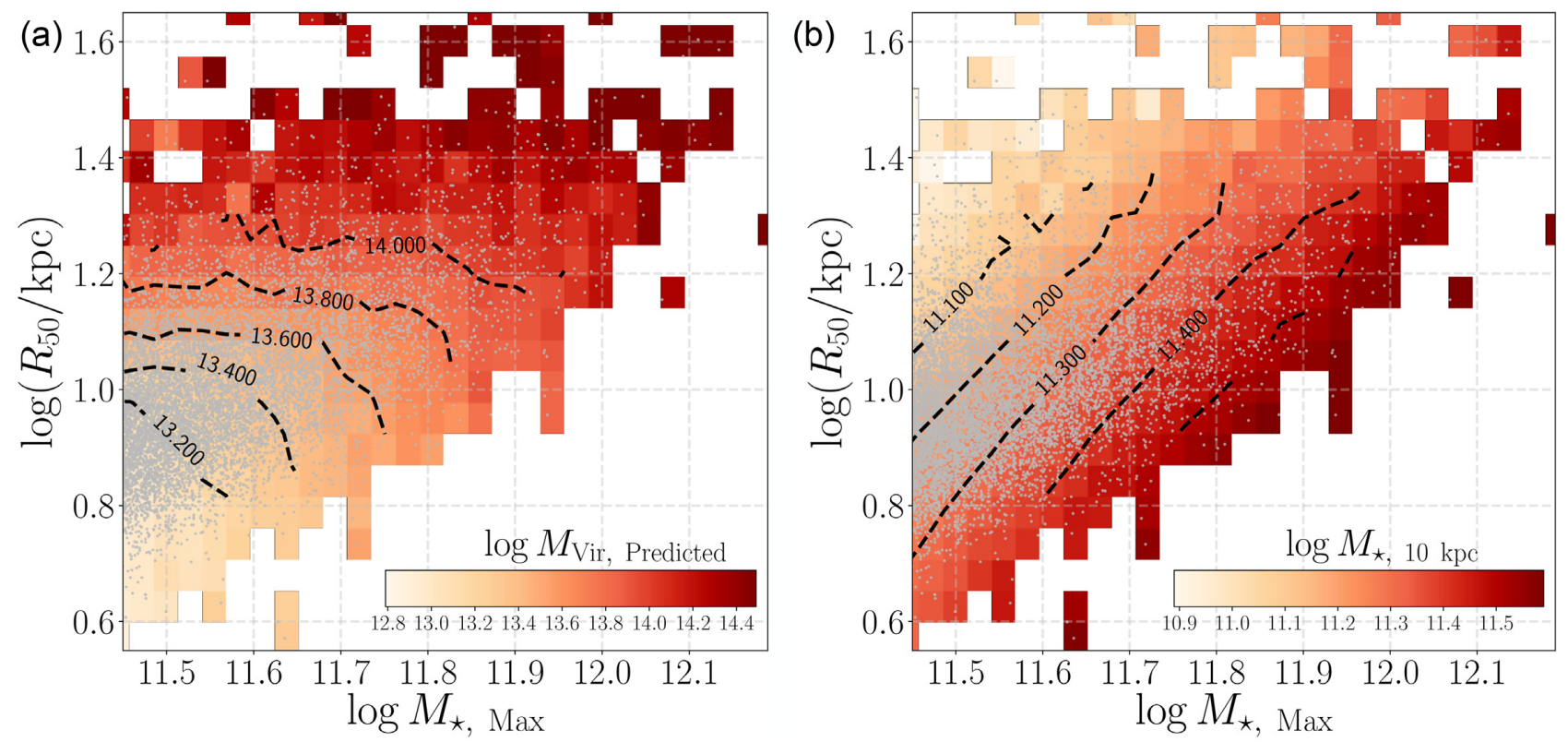

Figure 9. Stellar mass $\left(M_{\star}^{\max }\right)$-galaxy size $\left(R_{50}\right.$ measured using the observed 1D profile) relation colour coded by the predicted halo mass $\left(M_{\mathrm{vir}}\right)$ from the best-fitting model (a) and the stellar mass within $10 \mathrm{kpc}$ (b). For each HSC galaxy, we assign a $M_{\mathrm{vir}}$ using the best-fitting $M_{\star}^{\max }-M_{\star}^{10}-M_{\mathrm{vir}}$ relation. The Jupyter notebook for this figure is available here: https://github.com/dr-guangtou/asap/blob/master/note/fig9.ipynb.

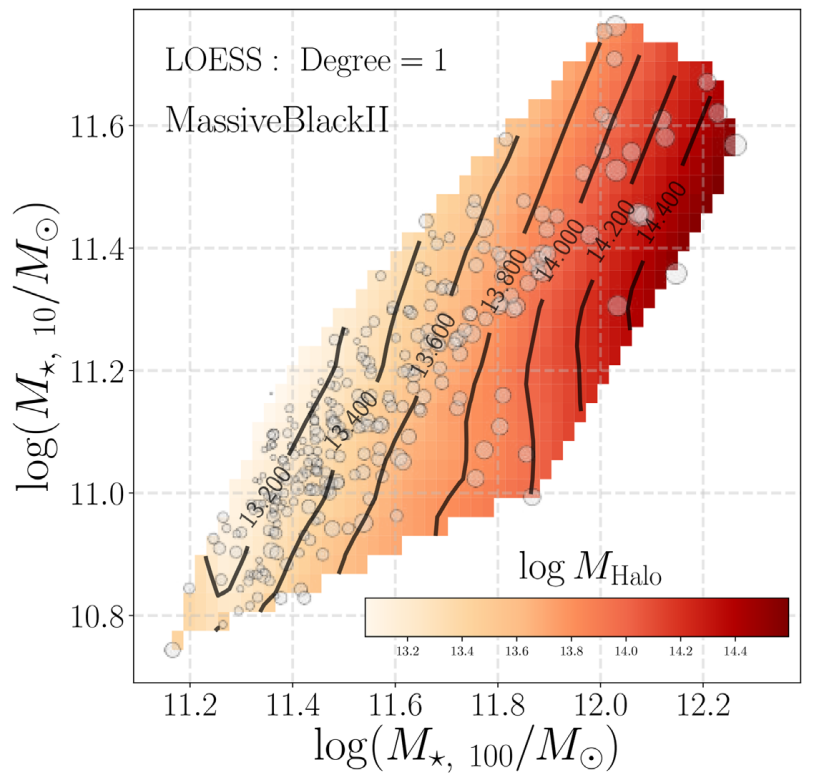

Figure 10. The $M_{\star}^{100}$ and $M_{\star}^{10}$ of massive galaxies from the MassiveBlackII simulation using randomly projected $2 \mathrm{D}$ stellar mass distributions. The plot shows their distributions over the aperture mass plane colour coded by halo mass. The density plot shows the halo mass trend recovered by the LOESS smoothing method. The Jupyter notebook for this figure is available here: https://github.com/dr-guangtou/asap/blob /master/note/fig10.ipynb.

$M_{\text {vir }}$ curves appears to be steeper than those of our best-fitting model (weaker correlation between $M_{\star}^{10}$ and $M_{\text {vir }}$ ), we see a general trend that is similar to our result: at fixed $M_{\star}^{100}$, the ones with lower $M_{\star}^{10}$ on average live in more massive dark matter haloes.

Currently, comparison with simulation is limited by the volume of high-resolution hydrosimulations and their capabilities to reproduce realistic massive galaxies. The SMF of massive galaxies using
$M_{\star}^{100}$ and the stellar mass density profiles of massive galaxies MassiveBlackII do show differences compared with the HSC observations (see Ardilla et al. in preparation). None the less, we consider this to be a valuable test and we will further investigate the robustness of this trend using other hydrosimulations in future work.

\subsection{Prediction of halo mass}

Our ASAP model suggests that, by including information about the stellar mass distribution (e.g. two-aperture stellar masses), one can build better proxies of halo mass. We test this potential by predicting the halo masses of massive clusters from the Cluster Lensing And Supernova survey with Hubble (CLASH) clusters (e.g. Postman et al. 2012) using only the photometry of their BCGs. DeMaio et al. (2018) conducted a careful study of the BCG+ICL of 23 CLASH clusters $\left(0.3<z<0.9 ; 3 \times 10^{13}<M_{500 c} / \mathrm{M}_{\odot}\right.$ $<9 \times 10^{14}$ ) using multiband, high-resolution HST Wide Field Camera 3 (WFC3) images. These authors derive surface brightness and colour profiles of these BCG+ICL to $r>100 \mathrm{kpc}$, along with stellar mass within 10 and 100 circular apertures using SED fitting. We ignore the differences caused by circular and elliptical apertures here and increase their $100 \mathrm{kpc}$ aperture mass by +0.05 dex to simulate our $M_{\star}^{\text {max }}$ measurement (see Paper I). After converting their aperture masses to the same cosmology and stellar population model ${ }^{15}$ used here, we predict the $M_{\text {vir }}$ of these BCGs using our bestfitting model. The CLASH sample includes mostly very massive clusters that host BCGs that are on average more massive than the HSC sample (panel a of Fig. 11).

Fig. 11 shows halo masses predicted both by the average $M_{\star}^{\max }$ $M_{\text {vir }}$ relation shown in Fig. 5 (green dots) and by the best-fitting

\footnotetext{
${ }^{15}$ DeMaio et al. (2018) uses the BC03 stellar population model. Based on tests from Paper I, we add a +0.1-dex empirical correction to the Flexible Stellar Population Synthesis (FSPS) mode used in this work.
} 

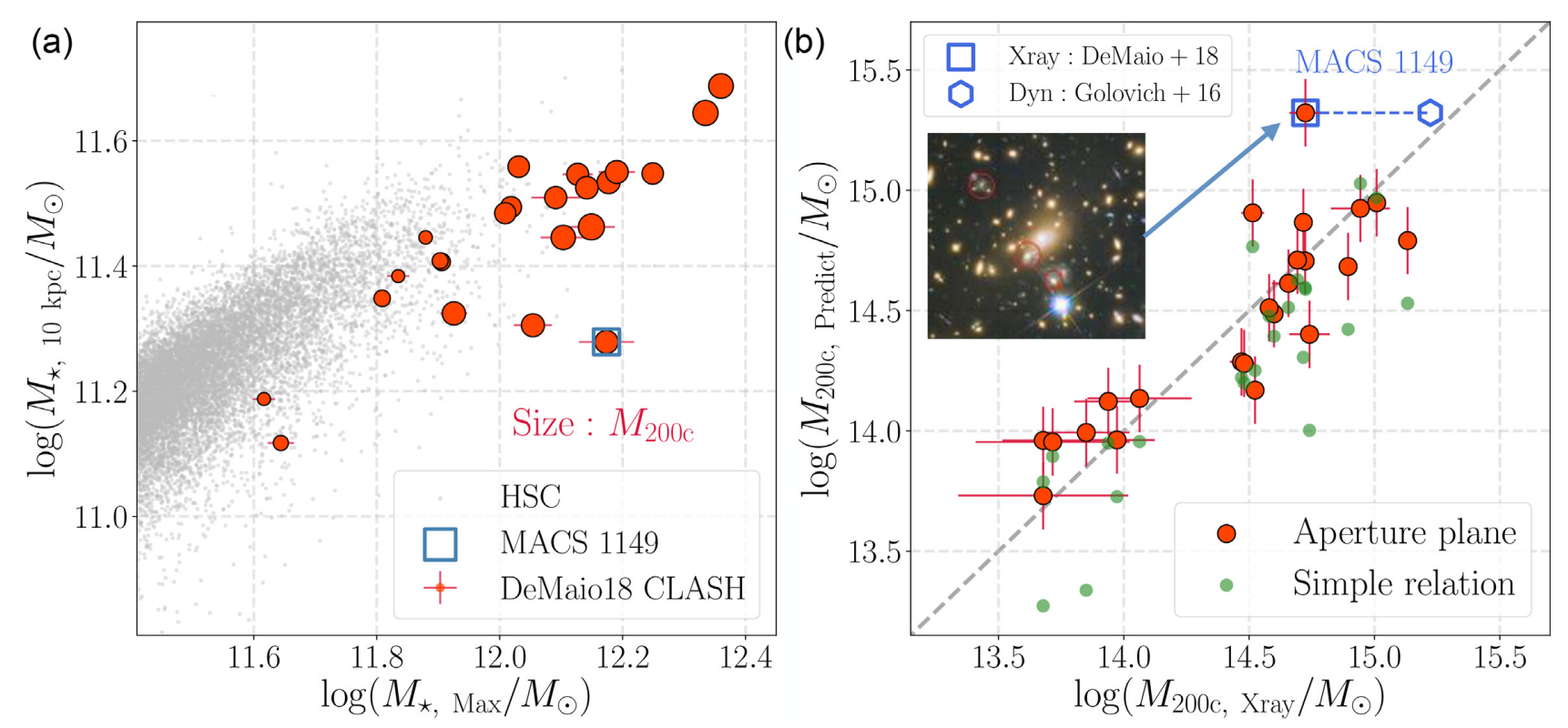

Figure 11. (a) Distribution of CLASH BCGs in the aperture mass plane. The stellar masses are based on Hubble Space Telescope (HST) observations (DeMaio et al. 2018; red points), where the symbol size indicates halo mass $\left(M_{200 c}\right)$ estimated from X-ray observations. (b) Comparison between our predicted $M_{200 c}$ values to those based on X-ray observations. Green points are predictions based on the $M_{\star}^{\max }-M_{\text {vir }}$ relation, whereas red points are based on the best-fitting aperture mass plane. The dashed line shows the one-to-one relation. The prediction using the aperture mass plane shows a tighter relation compared to X-ray masses. The outlier system, MACS1149, is highlighted using a blue box. We also show the coloured HST image of the BCG of MACS1149. The blue hexagon shows the value of $M_{200 c}$ as measured via dynamics (Golovich et al. 2016). The Jupyter notebook for this figure is available here: https://github.com/dr-guangtou/asap/blob/master/note/fig11.ipynb.

$M_{\star}^{\max }-M_{\star}^{10}-M_{\text {vir }}$ relation (red circle). In DeMaio et al. (2018), halo mass is measured using X-ray observations (e.g. Vikhlinin et al. 2009) and is defined as $M_{500 c}$. Using empirical relations from Diemer, More \& Kravtsov (2013), Diemer \& Kravtsov (2015), and the Colossus python package (Diemer 2017; code available here: https://bitbucket.org/bdiemer/colossus), we convert both the $M_{500 c}$ in DeMaio et al. (2018) and the $M_{\text {vir }}$ from our model to $M_{200 c}$. It is encouraging to see that the predicted halo mass values show good consistency with those based on X-rays. The values predicted using $M_{\star}^{\text {max }}$ alone show larger scatter compared to the X-ray mass estimates. This provides further evidence for one of the key findings of the present work: two-aperture stellar masses can be used to build better proxies of halo mass relative to models using $M_{\star}^{\max }$ alone. There is one BCG that shows a large offset (highlighted in both panels of Fig. 11) from the mean relations. The BCG belongs to the famous cluster MACS $1149+22$ at $z=0.544$ (see the inset picture) that gifted us multiple images of a highly magnified supernova (e.g. Kelly et al. 2015) and a $z \sim 9.1$ galaxy (e.g. Hashimoto et al. 2018). The region around the BCG is complex and partially overlaps with an image of a background star-forming galaxy. We suspect that the accuracy of photometry and $M_{\star} / L_{\star}$ estimation are affected by the complexity of extracting photometry for this system. Meanwhile, Golovich et al. (2016) estimate the halo mass of MACS $1149+22$ using dynamics of cluster members. The dynamics-based $M_{200 c}$ is higher than the X-ray value and is closer to our prediction. Although X-ray observation is often considered a good approach to measure cluster mass, hydrosimulations suggest that the hydrostatic equilibrium assumption used in the measurement can bias the cluster mass low by 10-30 per cent along with the projection effect (Evrard 1990; Nagai, Kravtsov \& Vikhlinin 2007; Mahdavi et al. 2008; Battaglia et al. 2012; Nelson, Lau \& Nagai 2014). Weak lensing measurements of cluster mass provide a way to constrain such bias. But previous works still show different results on this topic, from almost no bias ( $<10$ per cent level; e.g. Gruen et al. 2014; Israel et al. 2014; von der Linden et al. 2014; Applegate et al. 2016), to moderate level of bias (10-20 percent level; e.g. Donahue et al. 2014), and to bias larger than 20 percent (e.g. Okabe \& Smith 2016; Simet et al. 2017). And in Umetsu et al. (2014), the authors measure weak lensing halo mass for some of the same massive CLASH clusters used in DeMaio et al. (2018) and find noticeably more massive values than the X-ray ones. However, weak lensing cluster mass also suffers from systematics like the sub-structures in the halo and the projection effect. (e.g. Becker \& Kravtsov 2011; Meneghetti et al. 2011; van Uitert et al. 2012). Further discussion on this issue is beyond the scope of this paper, but it is worth investigating in the future to further improve our predictions of halo mass using aperture stellar masses.

\subsection{The fraction of $e x$ situ stars in massive galaxies}

Fig. 8 shows that the ex situ fraction predicted by our model and its relation with both stellar and halo mass are reasonable and are qualitatively consistent with hydrosimulation (e.g. TNG300). We now discuss two points in Fig. 8 of noteworthy interest. First, the large scatter in ex situ fractions at fixed stellar or halo mass suggests a small population of massive galaxies with low-ex situ fractions ( $<25$ percent). This special population could experience fewer mergers (especially major mergers) and is an interesting sample to study in greater detail. On panel (a) in Fig. 12, we colour code the $M_{\text {vir }}-f_{\text {exs }}$ relation for massive galaxies $\left(\log _{10}\left(M_{\star, \max } / \mathrm{M}_{\odot}\right) \geq 11.5\right)$ in our best-fitting model using the redshift of the last major halo merger (halo mass ratio larger than 1:3) extracted from the merger 

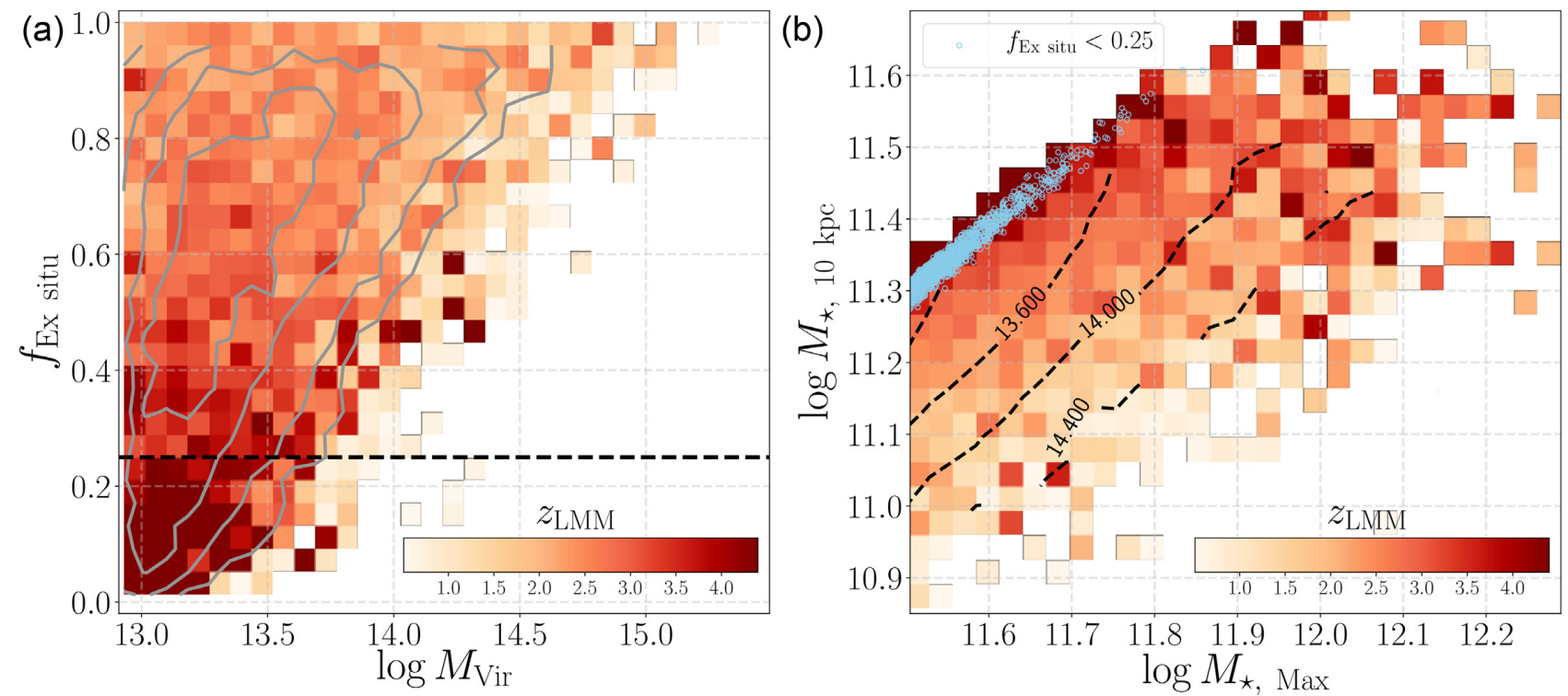

Figure 12. (a) Relation between the halo mass $\left(M_{\text {vir }}\right)$ and the fraction of ex situ stars for massive galaxies $\left(\log _{10}\left(M_{\star, \max } / \mathrm{M}_{\odot}\right) \geq 11.5\right)$ colour coded by the redshift of last major-merger ( $z_{\text {LMM }}$; halo mass ratio larger than 1:3) in the UniverseMachine model. The grey contours outline the number density distribution of model galaxies. A horizontal dashed line highlights the 25 percent limit that is used to define massive galaxies with low ex situ fraction. (b) The aperture mass plane for massive galaxies from the best-fitting model colour coded by $z_{\text {LMM }}$. Grey contours here indicate the iso- $M_{\mathrm{vir}}$ lines. The small population of massive model galaxies with $f_{\mathrm{exs}}<0.25$ is highlighted with light-blue dots. The Jupyter notebook for this figure is available here: https://github.com/dr-guangtou/asap/blob/master/note/fig12.ipynb.

trees of SMDPL haloes. We find that massive galaxies with low-ex situ fraction tend to live in relatively low-mass haloes and have not experienced major-mergers in the last 10 Gyrs, putting them among the oldest massive haloes in the $\mathrm{U}$

niverse. This small $(\sim 9$ per cent of massive galaxies with 11.5 $\left.<\log _{10}\left(M_{\star, \max } / \mathrm{M}_{\odot}\right) \geq 11.7\right)$ population locates exclusively on the upper edge of the aperture mass plane (panel b of Fig. 12). Such a special location suggests that they are much more compact than the similarly massive ones with a richer merging history. If haloes with such a unique assembly history are not artefacts of the UniverseMachine model, they could be very useful for studying galaxy assembly bias (e.g. Cooper et al. 2010; Wang et al. 2013; Zentner, Hearin \& van den Bosch 2014; Lin et al. 2016) or for providing a template of the distribution of in situ stars in massive haloes. The galaxies discussed here would be somewhat different in nature than 'relic' galaxies ${ }^{16}$ (e.g. Trujillo et al. 2014; Peralta de Arriba et al. 2016; Ferré-Mateu et al. 2017; Y1ldırım et al. 2017): the galaxies discussed here are more massive than typical relic galaxies, are larger in size, and are unaffected by stripping as this sample is predominantly centrals. Secondly, both the ASAP model and hydrosimulations predict a high ex situ fraction in the inner regions of massive galaxies. This is easy to understand given the current definition of ex situ stars. This is commonly defined as all the stars that are formed outside the halo of the main progenitor . The ex situ component therefore includes stars that were accreted from major mergers at very high redshift (e.g. $z>2$ ). Although this is a straightforward definition, it may not be the best choice to relate to observational studies of the stellar assembly history of massive galaxies for two reasons. First, it makes the ex situ component

\footnotetext{
${ }^{16}$ Typically defined as nearby compact quiescent galaxies with stellar mass and effective radius similar to the quiescent galaxies at high redshift. They often have steep inner stellar mass density profile and high central stellar velocity dispersion.
}

heterogeneous since ex situ stars can be formed at very different epochs and in haloes with a wide range of $M_{\text {vir }}$. Secondly, it is hard to separate the in situ and ex situ stars in the inner regions of massive galaxies because stars in both components are assembled at a very early time and share similar stellar population and kinematic properties. Although it is beyond the scope of this work, we argue that it may be worth considering alternative and potentially more instructive decompositions for massive galaxies.

\section{SUMMARY AND CONCLUSIONS}

Using data from the HSC survey, we perform careful aperture mass and weak lensing measurements for a sample of $~ 3200$ $\log _{10}\left(M_{\star, \max } / \mathrm{M}_{\odot}\right)>11.6$ massive galaxies. Using weak lensing, we reveal a tight connection between the stellar mass distribution of massive central galaxies and their total dark matter halo mass. At fixed 'total' stellar mass $\left(M_{\star}^{\max }\right)$, massive galaxies with more extended mass distributions tend to live in more massive dark matter haloes. This provides a new independent confirmation, backed by direct weak lensing measurements, of the results from Paper II that $M_{\text {vir }}$ varies systematically over the aperture $M_{\star}$ plane. To model both the weak lensing and the aperture SMFs, we build a full forward model based on a special version of the semiempirical model UniverseMachine and the SMDPL simulation. UniverseMachine leverages the ability of high-resolution simulations to identify and track the full merger history of dark matter haloes; using UniverseMachine as the bedrock of our model allows us to study the co-evolution of massive galaxies and their haloes. We augment the baseline UniverseMachine model with two prescriptions that allow us to fit HSC data and predict aperture masses. Our model makes the two following assumptions. We assume (1) a tight correlation between halo mass and the mass of its entire stellar content (TSHMR) and (2) a certain fraction of the in situ and ex situ stars locate within the inner $10 \mathrm{kpc}$ of 
massive galaxies. In our model, the well-studied SHMR and its scatter emerge from the TSHMR. We show that this model provides an excellent description of the observed SMFs for $M_{\star}^{\max }$ and $M_{\star}^{10}$, as well as the $\Delta \Sigma$ profiles in a series of $M_{\star}^{\max }-M_{\star}^{10}$ bins. The main conclusions from the current best-fitting model include the following:

(i) $M_{\text {vir }}$ varies systematically over the aperture mass plane. The iso- $M_{200 \mathrm{~b}}$ curves run almost parallel with the direction of the $M_{\star}^{\max }$ $M_{\star}^{10}$ relation. The model confirms that at fixed $M_{\star}^{\max }$, galaxies with more extended stellar mass distributions (lower $M_{\star}^{10}$ or larger size) live in more massive dark matter haloes. It also shows that scatter in $M_{\text {vir }}$ at either fixed $M_{\star}^{\max }$ or $M_{\star}^{10}$ is quite large.

(ii) The above trends can be summarized into a simple $M_{\star}^{\max }$ $M_{\star}^{10}-M_{\text {vir }}$ relation that provides a tighter connection with halo mass than $M_{\star}^{\max }$ alone.

(iii) The usage of two aperture masses can help reduce the scatter in halo mass at fixed total stellar mass. While the standard SHMR in the form of $\left\langle M_{\mathrm{vir}}\right\rangle_{M_{\star}}$ typically shows scatter in halo mass of $\sim 0.25$ dex at $11.5<\log M_{\star}^{\max }$, this scatter can be reduced to the $\sim 0.15$ dex level by utilizing our results based on $M_{\star}^{\max }-M_{\star}^{10}-M_{\text {vir }}$ scaling relation.

(iv) Our model predicts that the ex situ fraction increases with both the stellar and halo mass; and it shows that the ex situ component even dominates the inner $10 \mathrm{kpc}$ of massive galaxies. These predictions are consistent with results from the TNG simulations.

Our results strongly suggest that information about the assembly history of massive dark matter haloes is encoded in the stellar mass distributions of their massive central galaxies. This opens a new window for studying the assembly histories of group- and clustermass haloes by using carefully derived proxies based on massive galaxy profiles.

\section{ACKNOWLEDGEMENTS}

The authors would like to thank Frank van den Bosch, Sandra Faber, Joel Primack for useful discussions and suggestions. This material is based upon work supported by the National Science Foundation under Grant No. 1714610. The authors acknowledge support from the Kavli Institute for Theoretical Physics. This research was also supported in part by National Science Foundation under Grant No. NSF PHY11-25915 and Grant No. NSF PHY1748958. AL acknowledges support from the David and Lucille Packard foundation, and from the Alfred P Sloan foundation.

The Hyper Suprime-Cam (HSC) collaboration includes the astronomical communities of Japan and Taiwan, and Princeton University. The HSC instrumentation and software were developed by National Astronomical Observatory of Japan (NAOJ), Kavli Institute for the Physics and Mathematics of the Universe (Kavli IPMU), University of Tokyo, High Energy Accelerator Research Organization (KEK), Academia Sinica Institute for Astronomy and Astrophysics in Taiwan (ASIAA), and Princeton University. Funding was contributed by the FIRST program from Japanese Cabinet Office, Ministry of Education, Culture, Sports, Science and Technology (MEXT), Japan Society for the Promotion of Science (JSPS), Japan Science and Technology Agency (JST), Toray Science Foundation, NAOJ, Kavli IPMU, KEK, ASIAA, and Princeton University. Funding for Sloan Digital Sky SurveyIII (SDSS-III) has been provided by Alfred P. Sloan Foundation, the Participating Institutions, National Science Foundation, and U.S. Department of Energy. The SDSS-III website is http://ww w.sdss3.org. SDSS-III is managed by the Astrophysical Research
Consortium for the Participating Institutions of the SDSS-III Collaboration, including University of Arizona, the Brazilian Participation Group, Brookhaven National Laboratory, University of Cambridge, University of Florida, the French Participation Group, the German Participation Group, Instituto de Astrofisica de Canarias, the Michigan State/Notre Dame/JINA Participation Group, Johns Hopkins University, Lawrence Berkeley National Laboratory, Max Planck Institute for Astrophysics, New Mexico State University, New York University, Ohio State University, Pennsylvania State University, University of Portsmouth, Princeton University, the Spanish Participation Group, University of Tokyo, University of Utah, Vanderbilt University, University of Virginia, University of Washington, and Yale University. The Pan-STARRS1 surveys (PS1) have been made possible through contributions of Institute for Astronomy; University of Hawaii; the Pan-STARRS Project Office; the Max-Planck Society and its participating institutes: the Max Planck Institute for Astronomy, Heidelberg, and the Max Planck Institute for Extraterrestrial Physics, Garching; Johns Hopkins University; Durham University; University of Edinburgh; Queen's University Belfast; Harvard-Smithsonian Center for Astrophysics; Las Cumbres Observatory Global Telescope Network Incorporated; National Central University of Taiwan; Space Telescope Science Institute; National Aeronautics and Space Administration under Grant No. NNX08AR22G issued through the Planetary Science Division of the National Aeronautics and Space Administration (NASA) Science Mission Directorate; National Science Foundation under Grant No. AST-1238877; University of Maryland, and Eotvos Lorand University. This research makes use of software developed for the Large Synoptic Survey Telescope. We thank the LSST project for making their code available as free software at http: //dm.lsstcorp.org. The CosmoSim database used in this research is a service by the Leibniz-Institute for Astrophysics Potsdam (AIP). The MultiDark database was developed in cooperation with the Spanish MultiDark Consolider Project CSD2009-00064. This research made use of: STSCI_PYTHON, a general astronomical data analysis infrastructure in Python. STSCI_PYTHON is a product of the Space Telescope Science Institute, which is operated for NASA by Association of Universities for Research in Astronomy (AURA); SciPy, an open source scientific tool for Python (Jones et al. 2001); NumPy, a fundamental package for scientific computing with Python (Walt, Colbert \& Varoquaux 2011); Matplotlib, a 2D plotting library for Python (Hunter 2007); Ast ropy, a communitydeveloped core Python package for astronomy (Astropy Collaboration et al. 2013); scikit-learn, a machine-learning library in Python (Pedregosa et al. 2011); I Python, an interactive computing system for Python (Pérez \& Granger 2007); sep Source Extraction and Photometry in Python (Barbary et al. 2015); palettable, colour palettes for Python; emcee, Seriously Kick-Ass MCMC in Python; Colossus, COsmology, haLO and large-Scale StrUcture toolS (Diemer 2015).

\section{REFERENCES}

Abazajian K. N. et al., 2009, ApJS, 182, 543

Aihara H. et al., 2011, ApJS, 193, 29

Aihara H. et al., 2018a, PASJ, 70, S4

Aihara H. et al., 2018b, PASJ, 70, S8

Alam S. et al., 2015, ApJS, 219, 12

Amon A. et al., 2018, MNRAS, 477, 4285

Applegate D. E. et al., 2016, MNRAS, 457, 1522

Astropy Collaboration, 2013, A\&A, 558, A33 
Axelrod T., Kantor J., Lupton R. H., Pierfederici F., 2010, in Proc. SPIE, Vol. 774. Software and Cyberinfrastructure for Astronomy, p. 774015

Bahé Y. M. et al., 2017, MNRAS, 470, 4186

Baldry I. K. et al., 2018, MNRAS, 474, 3875

Barbary K., Boone K., Deil C., 2015, sep: v0.3.0. Available at: http://dx.doi .org/10.5281/zenodo.15669

Battaglia N., Bond J. R., Pfrommer C., Sievers J. L., 2012, ApJ, 758, 74

Becker M. R., 2015, preprint (arXiv:1507.03605)

Becker M. R., Kravtsov A. V., 2011, ApJ, 740, 25

Behroozi P. S., Wechsler R. H., Wu H.-Y., 2013a, ApJ, 762, 109

Behroozi P. S., Wechsler R. H., Conroy C., 2013b, ApJ, 770, 57

Behroozi P., Wechsler R. H., Hearin A. P., Conroy C., 2019, MNRAS, 488, 3143

Bender R., Kormendy J., Cornell M. E., Fisher D. B., 2015, ApJ, 807, 56

Benson A. J., 2014, MNRAS, 444, 2599

Benson A. J., 2017, MNRAS, 467, 3454

Benson A. J., Bower R., 2010, MNRAS, 405, 1573

Bernardi M., Meert A., Sheth R. K., Vikram V., Huertas-Company M., Mei S., Shankar F., 2013, MNRAS, 436, 697

Bernardi M., Meert A., Vikram V., Huertas-Company M., Mei S., Shankar F., Sheth R. K., 2014, MNRAS, 443, 874

Bernardi M., Meert A., Sheth R. K., Fischer J.-L., Huertas-Company M., Maraston C., Shankar F., Vikram V., 2017, MNRAS, 467, 2217

Bezanson R., van Dokkum P. G., Tal T., Marchesini D., Kriek M., Franx M., Coppi P., 2009, ApJ, 697, 1290

Bosch J. et al., 2018, PASJ, 70, S5

Bradshaw C., Leauthaud A., Hearin A., Huang S., Behroozi P., 2019, preprint (arXiv:1905.09353)

Bryan G. L., Norman M. L., 1998, ApJ, 495, 80

Budzynski J. M., Koposov S. E., McCarthy I. G., Belokurov V., 2014, MNRAS, 437, 1362

Bundy K., Leauthaud A., Saito S., Maraston C., Wake D. A., Thomas D., 2017, ApJ, 851, 34

Cappellari M., 2014, Astrophysics Source Code Library, record ascl:1404 .001

Cappellari M. et al., 2013, MNRAS, 432, 1862

Chabrier G., 2003, PASP, 115, 763

Charlton P. J. L., Hudson M. J., Balogh M. L., Khatri S., 2017, MNRAS, 472, 2367

Clampitt J. et al., 2017, MNRAS, 465, 4204

Clauwens B., Hill A., Franx M., Schaye J., 2017, MNRAS, 469, L58

Cleveland W. S., Devlin S. J., 1988, J. Am. Stat. Assoc., 83, 596

Conroy C., Gunn J. E., 2010a, Astrophysics Source Code Library, record ascl: 1010.043

Conroy C., Gunn J. E., 2010b, ApJ, 712, 833

Conroy C., van Dokkum P. G., Kravtsov A., 2015, ApJ, 803, 77

Cooper M. C., Gallazzi A., Newman J. A., Yan R., 2010, MNRAS, 402, 1942

Cooper A. P., D’Souza R., Kauffmann G., Wang J., Boylan-Kolchin M., Guo Q., Frenk C. S., White S. D. M., 2013, MNRAS, 434, 3348

Coupon J. et al., 2015, MNRAS, 449, 1352

Coupon J., Czakon N., Bosch J., Komiyama Y., Medezinski E., Miyazaki S., Oguri M., 2018, PASJ, 70, S7

Crain R. A. et al., 2015, MNRAS, 450, 1937

Croton D. J. et al., 2016, ApJS, 222, 22

Damjanov I. et al., 2009, ApJ, 695, 101

Dawson K. S. et al., 2013, AJ, 145, 10

De Lucia G., Blaizot J., 2007, MNRAS, 375, 2

DeMaio T., Gonzalez A. H., Zabludoff A., Zaritsky D., Connor T., Donahue M., Mulchaey J. S., 2018, MNRAS, 474, 3009

Diemer B., 2015, Astrophysics Source Code Library, record ascl:1501.016

Diemer B., 2017, ApJS, 231, 5

Diemer B., Kravtsov A. V., 2015, ApJ, 799, 108

Diemer B., More S., Kravtsov A. V., 2013, ApJ, 766, 25

Donahue M. et al., 2014, ApJ, 794, 136

Dose V., 2003, Rep. Prog. Phys., 66, 1421

Driver S. P. et al., 2009, Astron Geophys., 50, 5.12

Driver S. P. et al., 2011, MNRAS, 413, 971
Evrard A. E., 1990, ApJ, 363, 349

Ferré-Mateu A., Trujillo I., Martín-Navarro I., Vazdekis A., Mezcua M., Balcells M., Domínguez L., 2017, MNRAS, 467, 1929

Foreman-Mackey D., Hogg D. W., Lang D., Goodman J., 2013, PASP, 125, 306

Genel S., Genzel R., Bouché N., Naab T., Sternberg A., 2009, ApJ, 701, 2002

Genel S. et al., 2014, MNRAS, 445, 175

Giodini S. et al., 2009, ApJ, 703, 982

Golovich N., Dawson W. A., Wittman D., Ogrean G., van Weeren R., Bonafede A., 2016, ApJ, 831, 110

Gruen D. et al., 2014, MNRAS, 442, 1507

Gu M., Conroy C., Behroozi P., 2016, ApJ, 833, 2

Guo Q. et al., 2011, MNRAS, 413, 101

Hahn O., Martizzi D., Wu H.-Y., Evrard A. E., Teyssier R., Wechsler R. H., 2017, MNRAS, 470, 166

Hashimoto T. et al., 2018, Nature, 557, 392

Hearin A. P. et al., 2017, AJ, 154, 190

Henden N. A., Puchwein E., Shen S., Sijacki D., 2018, MNRAS, 479, 5385

Henriques B. M. B., White S. D. M., Thomas P. A., Angulo R., Guo Q., Lemson G., Springel V., Overzier R., 2015, MNRAS, 451, 2663

Hill A. R. et al., 2017, ApJ, 837, 147

Hilz M., Naab T., Ostriker J. P., 2013, MNRAS, 429, 2924

Hirata C., Seljak U., 2003, MNRAS, 343, 459

Hoekstra H., 2007, MNRAS, 379, 317

Hopkins P. F., Cox T. J., Kereš D., Hernquist L., 2008, ApJS, 175, 390

Huang S., Ho L. C., Peng C. Y., Li Z.-Y., Barth A. J., 2013, ApJ, 768, L28

Huang S. et al., 2018a, MNRAS, 475, 3348

Huang S. et al., 2018b, PASJ, 70, S6

Huang S., Leauthaud A., Greene J. E., Bundy K., Lin Y.-T., Tanaka M., Miyazaki S., Komiyama Y., 2018c, MNRAS, 475, 3348

Hunter J. D., 2007, Comput. Sci. Eng., 9, 90

Israel H., Reiprich T. H., Erben T., Massey R. J., Sarazin C. L., Schneider P., Vikhlinin A., 2014, A\&A, 564, A129

Johansson P. H., Naab T., Ostriker J. P., 2009, ApJ, 697, L38

Jones E. et al., 2001, SciPy: Open source scientific tools for Python, Available at: http://www.scipy.org/

Jurić M. et al., 2017, ASPC, 279, 512

Kelly P. L. et al., 2015, Science, 347, 1123

Kelson D. D., Zabludoff A. I., Williams K. A., Trager S. C., Mulchaey J. S., Bolte M., 2002, ApJ, 576, 720

Khandai N., Di Matteo T., Croft R., Wilkins S., Feng Y., Tucker E., DeGraf C., Liu M.-S., 2015, MNRAS, 450, 1349

Kravtsov A. V., 2013, ApJ, 764, L31

Kravtsov A. V., Vikhlinin A. A., Meshcheryakov A. V., 2018, Astron. Lett., 44,8

Leauthaud A. et al., 2012a, ApJ, 744, 159

Leauthaud A. et al., 2012b, ApJ, 746, 95

Leauthaud A. et al., 2017, MNRAS, 467, 3024

Leja J., Johnson B. D., Conroy C., van Dokkum P. G., Byler N., 2017, ApJ, 837, 170

Lin Y.-T., Mohr J. J., 2004, ApJ, 617, 879

Lin Y.-T., Stanford S. A., Eisenhardt P. R. M., Vikhlinin A., Maughan B. J., Kravtsov A., 2012, ApJ, 745, L3

Lin Y.-T., Mandelbaum R., Huang Y.-H., Huang H.-J., Dalal N., Diemer B., Jian H.-Y., Kravtsov A., 2016, ApJ, 819, 119

Liske J. et al., 2015, MNRAS, 452, 2087

Longobardi A., Arnaboldi M., Gerhard O., Hanuschik R., 2015, A\&A, 579, A135

Lu Y., Mo H. J., Weinberg M. D., Katz N., 2011, MNRAS, 416, 1949

Lundgren B. F. et al., 2014, ApJ, 780, 34

Mahdavi A., Hoekstra H., Babul A., Henry J. P., 2008, MNRAS, 384, 1567

Mandelbaum R., Seljak U., Kauffmann G., Hirata C. M., Brinkmann J., 2006a, MNRAS, 368, 715

Mandelbaum R., Seljak U., Cool R. J., Blanton M., Hirata C. M., Brinkmann J., 2006b, MNRAS, 372, 758

Mandelbaum R. et al., 2018, MNRAS, 481, 3170 
Mandelbaum R. et al., 2018, PASJ, 70, S25

Mao Y.-Y., Zentner A. R., Wechsler R. H., 2018, MNRAS, 474, 5143

Medezinski E. et al., 2018, PASJ, 70, S28

Meneghetti M., Fedeli C., Zitrin A., Bartelmann M., Broadhurst T., Gottlöber S., Moscardini L., Yepes G., 2011, A\&A, 530, A17

Miyatake H. et al., 2019, ApJ, 875, 63

Miyazaki S. et al., 2012, McLean I. S., Ramsay S. K., Takami H., in Proc. SPIE Conf. Ser. Vol. 8446, Ground-based and Airborne Instrumentation for Astronomy IV. SPIE, Bellingham, p. $84460 Z$

More S., van den Bosch F. C., Cacciato M., Skibba R., Mo H. J., Yang X., 2011, MNRAS, 410, 210

Moster B. P., Naab T., White S. D. M., 2018, MNRAS, 477, 1822

Moustakas J. et al., 2013, ApJ, 767, 50

Muzzin A. et al., 2013, ApJ, 777, 18

Nagai D., Kravtsov A. V., Vikhlinin A., 2007, ApJ, 668, 1

Nelson K., Lau E. T., Nagai D., 2014, ApJ, 792, 25

Okabe N., Smith G. P., 2016, MNRAS, 461, 3794

Oke J. B., Gunn J. E., 1983, ApJ, 266, 713

Oogi T., Habe A., 2013, MNRAS, 428, 641

Oser L., Ostriker J. P., Naab T., Johansson P. H., Burkert A., 2010, ApJ, 725, 2312

Oser L., Naab T., Ostriker J. P., Johansson P. H., 2012, ApJ, 744, 63

Ownsworth J. R., Conselice C. J., Mortlock A., Hartley W. G., Almaini O., Duncan K., Mundy C. J., 2014, MNRAS, 445, 2198

Patel S. G. et al., 2013, ApJ, 766, 15

Patel S. G., Kelson D. D., Williams R. J., Mulchaey J. S., Dressler A., McCarthy P. J., Shectman S. A., 2015, ApJ, 799, L17

Pedregosa F. et al., 2011, J. Mach. Learn. Res., 12, 2825

Peralta de Arriba L., Quilis V., Trujillo I., Cebrián M., Balcells M., 2016, MNRAS, 461, 156

Pérez F., Granger B. E., 2007, Comput. Sci. Eng., 9, 21

Pillepich A. et al., 2018a, MNRAS, 473, 4077

Pillepich A. et al., 2018b, MNRAS, 475, 648

Postman M. et al., 2012, ApJS, 199, 25

Prat J. et al., 2018, PhRvD, 98, 042005

Qu Y. et al., 2017, MNRAS, 464, 1659

Reddick R. M., Wechsler R. H., Tinker J. L., Behroozi P. S., 2013, ApJ, 771, 30

Rodriguez-Gomez V. et al., 2016, MNRAS, 458, 2371

Rodríguez-Puebla A., Primack J. R., Avila-Reese V., Faber S. M., 2017, MNRAS, 470, 651

Roediger J. C., Courteau S., 2015, MNRAS, 452, 3209

Rozo E., Rykoff E. S., 2014, ApJ, 783, 80

Rykoff E. S. et al., 2014, ApJ, 785, 104

Schaye J. et al., 2015, MNRAS, 446, 521

Schlafly E. F., Finkbeiner D. P., 2011, ApJ, 737, 103

Shan H. et al., 2017, ApJ, 840, 104

Sharma S., 2017, ARA\&A, 55, 213

Shirasaki M., Takada M., Miyatake H., Takahashi R., Hamana T., Nishimichi T., Murata R., 2017, MNRAS, 470, 3476

Simet M., Battaglia N., Mandelbaum R., Seljak U., 2017, MNRAS, 466, 3663

Singh S., Mandelbaum R., Seljak U., Slosar A., Vazquez Gonzalez J., 2017, MNRAS, 471, 3827

Somerville R. S., Popping G., Trager S. C., 2015, MNRAS, 453, 4337

Sonnenfeld A., Wang W., Bahcall N., 2019, A\&A, 622, A30

Speagle J., et al., 2019, MNRAS, 490, 5658

Springel V., 2005, MNRAS, 364, 1105

Takada M., Hu W., 2013, Phys. Rev. D, 87, 123504

Tanaka M. et al., 2018, PASJ, 70, S9

Tenneti A., Mandelbaum R., Di Matteo T., Kiessling A., Khandai N., 2015, MNRAS, 453, 469

Tinker J. L. et al., 2017, ApJ, 839, 121

Toft S. et al., 2014, ApJ, 782, 68

Trujillo I., Ferré-Mateu A., Balcells M., Vazdekis A., Sánchez-Blázquez P., 2014, ApJ, 780, L20
Umetsu K. et al., 2014, ApJ, 795, 163

van den Bosch F. C., Ogiya G., 2018, MNRAS, 475, 4066

van den Bosch F. C., Ogiya G., Hahn O., Burkert A., 2018, MNRAS, 474, 3043

van der Burg R. F. J., Muzzin A., Hoekstra H., Wilson G., Lidman C., Yee H. K. C., 2014, A\&A, 561, A79

van der Wel A. et al., 2014, ApJ, 788, 28

van Dokkum P. G. et al., 2008, ApJ, 677, L5

van Dokkum P. G. et al., 2015, ApJ, 813, 23

van Uitert E., Hoekstra H., Schrabback T., Gilbank D. G., Gladders M. D.,

Yee H. K. C., 2012, A\&A, 545, A71

van Uitert E. et al., 2016, MNRAS, 459, 3251

Vikhlinin A. et al., 2009, ApJ, 692, 1033

Vogelsberger M. et al., 2014, MNRAS, 444, 1518

von der Linden A. et al., 2014, MNRAS, 439, 2

Vulcani B. et al., 2016, ApJ, 816, 86

Walt S. v. d., Colbert S. C., Varoquaux G., 2011, Comput. Sci. Eng., 13, 22

Wang L., Weinmann S. M., De Lucia G., Yang X., 2013, MNRAS, 433, 515

Wechsler R. H., Tinker J. L., 2018, ARA\&A, 56, 435

Wellons S. et al., 2016, MNRAS, 456, 1030

White S. D. M., Frenk C. S., 1991, ApJ, 379, 52

Yıldırım A., van den Bosch R. C. E., van de Ven G., Martín-Navarro I., Walsh J. L., Husemann B., Gültekin K., Gebhardt K., 2017, MNRAS, 468,4216

Yoon Y., Im M., Kim J.-W., 2017, ApJ, 834, 73

Zentner A. R., Hearin A. P., van den Bosch F. C., 2014, MNRAS, 443, 3044

Zhang Y. et al., 2019, ApJ, 874, 165

Ziparo F. et al., 2016, A\&A, 592, A9

Zu Y., Mandelbaum R., 2015, MNRAS, 454, 1161

Zu Y., Mandelbaum R., 2016, MNRAS, 457, 4360

\section{APPENDIX A: $M_{\text {VIR }}$ TRENDS OF KEY \\ PREDICTIONS IN THE UniverseMachine MODEL}

As explained in Section 5.3, besides $M_{\mathrm{vir}}$, ASAP model also relies on three key predictions from the special version of UniverseMachine model used in this work:

(i) $\delta_{\text {cen }}$ : the ratio between the stellar mass of central galaxy and the total stellar mass within the halo. This parameter reflects the 'dominance' of central galaxy in the halo. It is determined by the complex merger history of both the halo and the central galaxy.

(ii) $\delta_{\text {ins }}$ and $\delta_{\text {exs }}$ : the fractions of stellar mass in the in situ and ex situ components for each galaxy. They are determined by both the star formation and mass-assembly history of each galaxy in the halo.

On the left side of Fig A1, we show the relationship between $M_{\star}^{\text {all }}$ and $\delta_{\text {cen }}$. On average, central galaxy becomes less dominating in more massive haloes given the tight relation between $M_{\mathrm{vir}}$ and $M_{\star}^{\text {all }}$. Meanwhile, UniverseMachine model predicts a significant scatter of $\delta_{\text {cen }}$ at fixed $M_{\star}^{\text {all }}$, especially for less massive haloes. The scatter of $\delta_{\text {cen }}$ at fixed $M_{\star}^{\text {all }}$ dominates the scatter of the SHMR predicted by the ASAP model. Given the resolution of the $N$-body simulation used in UniverseMachine model and the adopted assumptions to handle galaxy mergers, it is possible that such large scatter of $\delta_{\text {cen }}$ is not very reliable. On the right side of Fig A1, we demonstrate that the in situ (ex situ) mass fraction rapidly decreases (increases) with $M_{\mathrm{vir}}$, which is consistent with results from recent hydrodynamic simulations. 

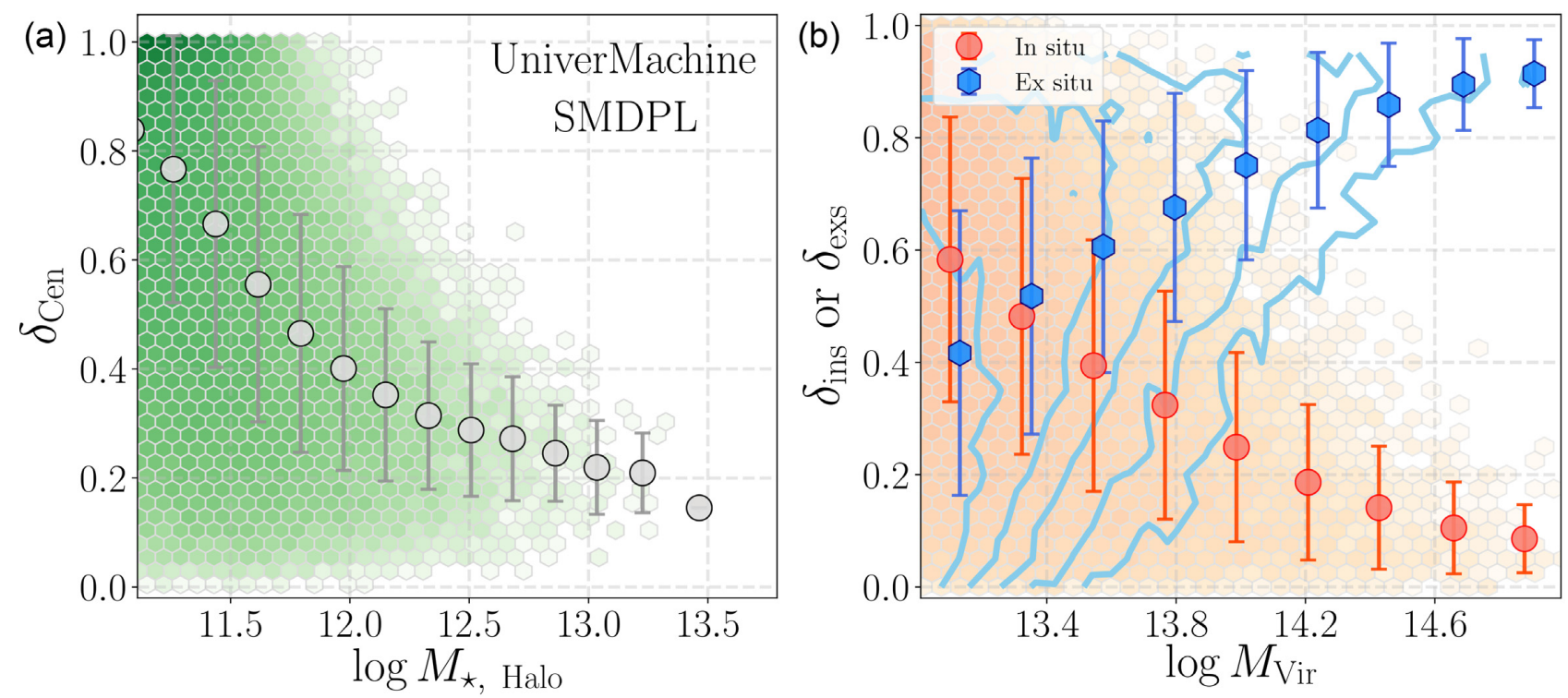

Figure A1. Distributions of $M_{\star}$ fraction of central galaxy $\left(M_{\star}^{\text {cen }} / M_{\star}^{\text {all }}\right)$ and its dependence on $M_{\text {vir }}$. Colour shows the number density of galaxies in log-scale. The median central mass fractions in a series of $M_{\mathrm{vir}}$ bins are highlighted using grey circles along with the $1 \sigma$ scatter in each bin. The shaded region on the left side is for the $M_{\text {vir }}$ range ignored in this work. The Jupyter notebook for this figure is available here: https://github.com/dr-guangtou/asap/blob/master/note/figA1.ipynb.

\section{APPENDIX B: PERFORMANCE OF MVIR ESTIMATORS}

As mentioned in Section 6.4, we attempt to assign $M_{\text {vir }}$ to massive galaxies in HSC surveys by comparing the observed aperture stellar masses to the ones predicted by the best-fittng ASAP model. Here, we briefly demonstrate the performances of two $M_{\text {vir }}$ estimators here: the random forest regressor and the $2 \mathrm{D} M_{\star}^{\max }-M_{\star}^{10}-M_{\mathrm{vir}}$ scaling relation. The Jupyter notebook used for $M_{\mathrm{vir}}$ predictions is available here: https://github.com/dr-guangtou/asap/blob/master/ note/assign_halo_mass.ipynb. For the random forest regressor, we use the RandomForestRegressor from the sciki-learn PYTHON package. We choose to use 20 estimators and mean absolute error criteria. We train the random forest regressor using the predicted $\mathcal{M}_{\star}^{\text {Max }}$ and $\mathcal{M}_{\star}^{10}$ of central galaxies from the bestfitting ASAP model, then validate it using a realization of the ASAP model with parameters slightly deviated from the best-fitting values. On the left side of Fig B1, we visualize the performance of this estimator across the aperture stellar mass plane. We choose to use $\left(\log M_{\mathrm{vir}}^{\text {predict }}-\log M_{\mathrm{vir}}^{\text {true }}\right) / \sigma_{\log M_{\mathrm{vir}}^{\text {true }}}$ to test the accuracy of the
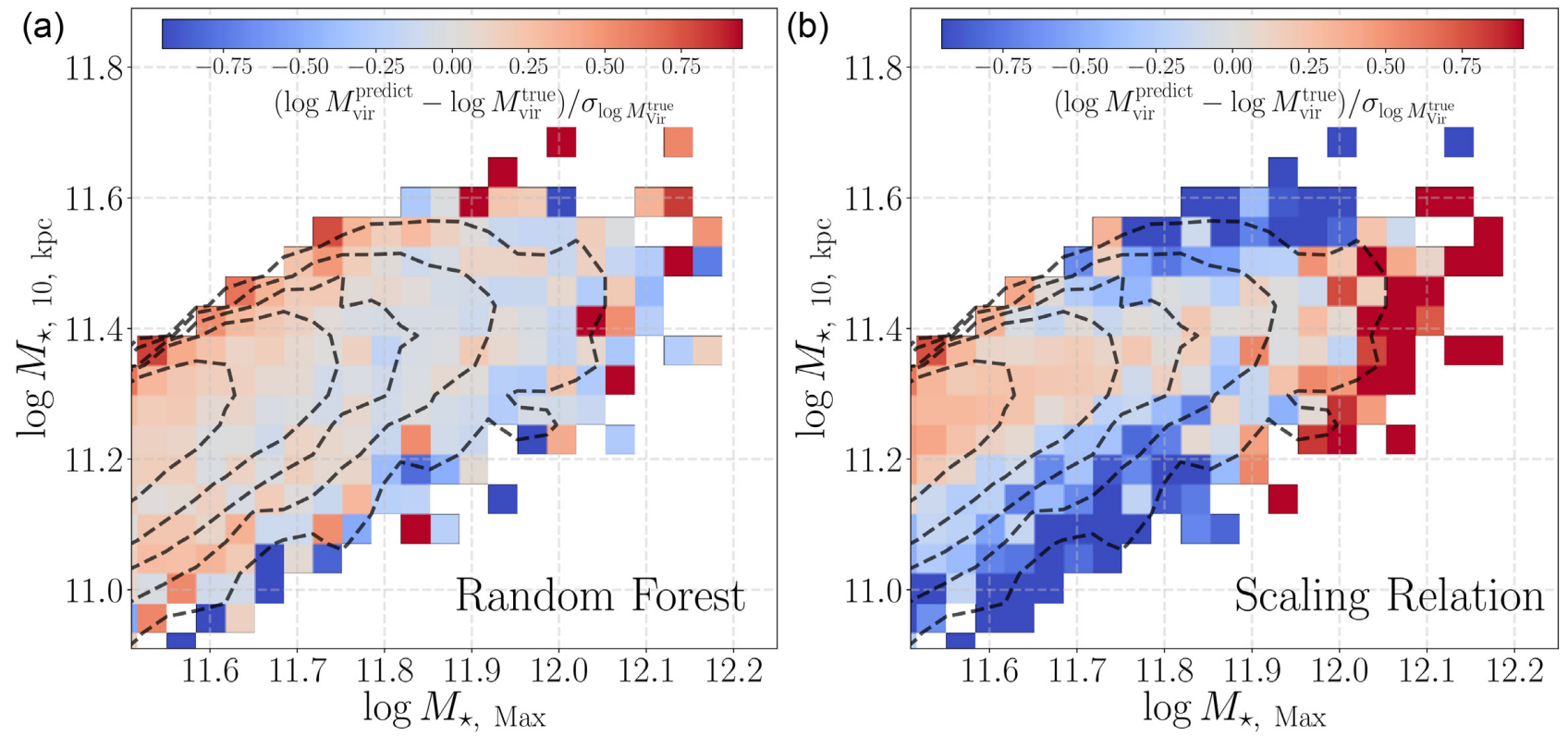

Figure B1. Evaluation of two different $M_{\text {vir }}$ predictors based on the halo mass trend over the aperture mass plane. The left-hand panel is for the random forest regressor and the right side is for the $M_{\star}^{\max }-M_{\star}^{10}-M_{\text {vir }}$ scaling relation. On both figures, the colour indicates the relative differences between the predicted $M_{\mathrm{vir}}$ and the true values from the UniverseMachine model. Regions occupied by most observed HSC galaxies are highlighted using grey contours. The Jupyter notebook for this figure is available here: https://github.com/dr-guangtou/asap/blob/master/note/figB1.ipynb. 
prediction where $\sigma_{\log M_{v i d}^{\text {true }}}$ is the scatter of $M_{\mathrm{vir}}$ in each 2D bin of aperture masses. As expected, the random forest regressor can easily capture the detailed trend of $M_{\text {vir }}$ over the 2D aperture stellar mass plane. Meanwhile, we have shown the best-fitting 2D $M_{\star}^{\max }$ $M_{\star}^{10}-M_{\text {vir }}$ scaling relation in Section 6.4. We visualize its accuracy on the right side of Fig B1. As one can see, this simple scaling relation can still capture the main $M_{\text {vir }}$ trend over the regions that are occupied by most HSC galaxies (highlighted by contours). Although the $M_{\text {vir }}$ predicted by this 2D scaling relation starts to show deviations compared to true values at the edges of the aperture stellar mass relation, the systematic differences are still comparable to the scatters of $M_{\mathrm{vir}}$ in these bins. In the next paper of this series, we will be looking for more reliable way to predict $M_{\text {vir }}$ based on the stellar mass distributions of massive galaxies and improved version of ASAP model. We will also directly test these predictions using HSC weak lensing calibrations.

This paper has been typeset from a $\mathrm{T}_{\mathrm{E}} \mathrm{X} / \mathrm{LT} \mathrm{E} \mathrm{X}$ file prepared by the author. 